\title{
Ambiguous Information, Risk Aversion, and Asset Pricing
}

\author{
Philipp Karl ILLEDITSCH*
}

February 14, 2009

\begin{abstract}
I study the effects of aversion to risk and ambiguity (uncertainty in the sense of Knight (1921)) on the value of the market portfolio when investors receive information that they find difficult to link to fundamentals and hence treat as ambiguous. Investors consider a set of models that consists of a single normally distributed marginal for fundamentals and a family of normally distributed conditionals that relate information to fundamentals. Hence, they neither know the posterior mean nor the posterior variance of fundamentals. I show that when investors receive ambiguous information, then the interpretation of this information can drastically change. This leads to a discontinuity in the equilibrium price of the market portfolio, excess volatility, negative skewness, and excess kurtosis of stock market returns. Moreover, a higher signal value does not always lead to a higher price.
\end{abstract}

\footnotetext{
${ }^{*}$ The Wharton School, University of Pennsylvania, 3620 Locust Walk, 2300 SH-DH, Philadelphia, PA 19104 - 6367, phone: 215 - 898 - 3477, e-mail: pille@wharton.upenn.edu. I would like to thank Kerry Back, Philip Bond, Domenico Cuoco, Michael Gallmeyer, Neal Galpin, Shane Johnson, Dmitry Livdan, Bryan Routledge, Francesco Sangiorgi, Martin Schneider, Robert Stambaugh, Semih Tartaroglu, Julie Wu, Amir Yaron, Motohiro Yogo, Stanley Zin, seminar participants at the Mays Business School, the Rotman School of Management, the Stern School of Business, the Wharton School, Carnegie Mellon, the University of Amsterdam, Tilburg University, Warwick Business School, NHH, and BI, and conference participants at the New Stars in Finance Conference in Madrid for helpful suggestions. I am also very grateful for the generous support of the Mays Business School during my Ph.D. education.
} 
News, as for instance the default of Lehman Brothers or the rescue of AIG, is often associated with an increase in stock market volatility. This excess sensitivity of prices to news about fundamentals is well documented in the literature and in general difficult to explain 1 In this paper I show that when investors are averse to risk and ambiguity (Knightian uncertainty) and receive information that they find difficult to link to fundamentals, then the interpretation of this information can drastically change. This leads to a discontinuity in the equilibrium price of the market portfolio and excess volatility of stock market returns.

Investors who receive a signal about economic fundamentals face ambiguity and risk when they evaluate an investment in the market portfolio because they neither know the model that links this signal to the market portfolio nor the value of the market portfolio given this signal. If investors are averse to ambiguity, they behave as if they have multiple models in mind when they process this signal. Specifically, they evaluate the outcome of an investment with respect to every model they think is plausible and then choose the model that leads to the lowest expected utility. These preferences are consistent with experimental evidence (Ellsberg (1961)) and have a solid axiomatic foundation: Gilboa and Schmeidler (1989) axiomatize this behavior in an atemporal setting and Epstein and Schneider (2003) generalize their work to a dynamic setting.

To study the effects of risk and ambiguity on the value of the market portfolio I consider a representative investor with CARA utility who receives a noisy signal about the aggregate dividend. I follow Epstein and Schneider (2008) and assume that the set of models that links this signal to the dividend consists of a single normal marginal and a family of normal conditionals. Hence, processing an ambiguous signal leads to ex-post ambiguity about the mean and the variance of the dividend even though there is no ex-ante ambiguity about the dividend.

I show that there is a discontinuity in the equilibrium signal-to-price map and a higher signal value does not always lead to a higher price. Specifically, the equilibrium stock price is unique except for a specific signal value at which there is an interval of equilibrium stock prices. If the signal increases through this critical point, then the price suddenly drops and hence better information leads to a lower stock price.

The stock price is not monotone in the signal in equilibrium because the model that

\footnotetext{
${ }^{1}$ Shiller (1981) and LeRoy and Porter (1981) among others document the excess volatility puzzle.
} 
leads to the lowest expected utility for the representative investor depends on the signal. Specifically, if the signal is bad, then it is treated as informative and thus the residual variance is low. Conversely, if the signal is good, then it is treated as uninformative and thus the residual variance is high. Hence, a bad signal leads to a low risk premium and thus to a high price.

There is an interval of equilibrium stock prices because the utility of the representative investor has a kink at the market clearing stock demand. This kink prevails when I aggregate over investors with different preferences as long as there is a common set of beliefs (models that link the signal to the dividend). Intuitively, if investors share a common set of beliefs, then there is an interval of prices at which they do not the change their position in the asset. All these prices are equilibrium prices when individual demands, which can differ in magnitude depending on an investor's risk aversion, add up to the market clearing demand. This result has the interesting consequence that speculation - which Billot, Chateuneuf, and Gilboa (2000) show occurs if and only if investors have no belief in common - smoothes out the discontinuity in the equilibrium-signal-to-price map and hence avoids drastic changes in the price of the market portfolio 2

When investors receive ambiguous information and anticipate ambiguous information in the future, then the equilibrium price is multi-valued for two critical signal values. Specifically, if the signal increases through the first critical value, then the price suddenly drops whereas if the price increases through the second critical value, then the price suddenly increases. Hence, learning about fundamentals over time increases the sensitivity of prices to ambiguous information and hence the variance and kurtosis of stock market returns. Moreover, the stock price variance conditional on bad news is always larger than the variance conditional on good news because the sudden drop for bad news is larger than the sudden increase for good news.

Ambiguous information affects an investor's utility through the realization of the current signal and the informativeness of the current and the future signal. If the current signal roughly confirms the unconditional mean of the dividend, then the marginal investor treats both signals as uninformative and hence the risk premium is larger than when the news are

\footnotetext{
${ }^{2}$ Investors with standard expected utility preferences engage in speculative trade or betting if and only if they have different beliefs about the informativeness of the signal.
} 
either good or bad. If one imagined the signal increasing through the first critical value (which is lower than the unconditional mean of the dividend), the risk premium of the asset would suddenly increase, whereas if one imagined the signal increasing through the second critical value (which is higher than the unconditional mean of the dividend), the risk premium would suddenly decrease.

This paper is most closely related to Epstein and Schneider (2008), who investigate the impact of ambiguous information on stock prices assuming a representative investor who is risk neutral and averse to ambiguous information. I extend their work along three dimension: (i) investors are risk averse ${ }^{3}$, (ii) investors are heterogenous, (iii) investors can learn from ambiguous signals over time 4 I show that risk aversion leads to qualitatively very different implications for the equilibrium signal-to-price map with and without learning over time, and it amplifies the effects of ambiguous information on the conditional distribution of stock market returns. Moreover, I prove the existence of a representative investor when investor have different risk aversion and discuss the properties of the equilibrium when investors also differ with respect to aversion to ambiguity.

This paper is also related to the literature on limited market participation. Specifically, Dow and Werlang (1992) show in a partial equilibrium framework, that there is a range of prices at which investors are neither long or short the asset. Cao, Wang, and Zhang (2005) extend their result and show that limited stock market participation can arise endogenously in equilibrium when investors differ with respect to their aversion to ambiguity. I show that when investor receive ambiguous information, then the worst case scenario belief depends on the asset demand and the signal. This leads to a demand function that is flat for two ranges of prices. Specifically, there is an interval of prices at which (i) investors don't participate in the market because they are ambiguous about the mean of the asset and (ii) investors don't change their long/short position in the asset because they are ambiguous about the mean and the risk premium of the asset.

Routledge and Zin (2001) and Caballero and Krishnamurthy (2008) study the connection of ambiguity with liquidity. Routledge and Zin (2001) consider a financial intermediary

\footnotetext{
${ }^{3}$ Epstein and Schneider (2008) consider an example in which the signal can take on two values and solve it numerically when investor are risk averse.

${ }^{4}$ Epstein and Schneider's dynamic model focuses on short learning episodes where investors receive one ambiguous signal about the next innovation in dividends whereas in this model investor receive and anticipate an ambiguous signal about a future liquidating dividend.
} 
who makes a market in a derivative security and show that ambiguity can drastically increase the bid-ask spread and hence reduce liquidity. Caballero and Krishnamurthy (2008) study the effects of ambiguity about the impact of aggregate liquidity shocks on investors and show that this ambiguity can lead to a socially inefficient flight to quality. In this paper ambiguous information does not have an effect on market liquidity but nevertheless leads to drastic changes in the price of the market portfolio and hence excess variance and kurtosis of stock market returns.

The fact that ambiguity aversion can lead to value functions that are not differentiable everywhere and hence to a continuum of equilibrium prices for some states of the world is not new. Epstein and Wang (1994) write in their abstract: "A noteworthy feature of the model is that uncertainty may lead to equilibria that are indeterminate, that is, there may exist a continuum of equilibria for given fundamentals." The striking result of this paper is that only the combination of risk aversion and ambiguity aversion leads to a price discontinuity and an interval of equilibria for a particular signal value when investors process ambiguous information. Hence, the effects of ambiguity aversion on stock prices can be distinguished from the effects of risk alone.

This paper is also related to the literature on learning about fundamentals with standard expected utility preferences to explain the excess volatility puzzle. Brennan and Xia (2001) and Veronesi (2000) consider an economy in which investors learn from dividends (and a noisy signal in Veronesi (2000)) about the unobservable expected dividend growth rate. Brennan and Xia (2001) assume that the expected dividend growth rate follows a mean reverting Ornstein-Uhlenbeck process whereas Veronesi (2000) assume that it follows an ndimensional regime switching process. In this paper investors are also averse to ambiguity which leads to drastic changes in the interpretation of news and hence excess sensitivity of prices to news about fundamentals.

This paper is also related to work on the equity premium puzzle, the risk-free rate puzzle, and the excess volatility puzzle by Maenhout (2004) and Leippold, Trojani, and Vanini (2008). Maenhout (2004) solves the dynamic portfolio choice problem of an investor with power utility and Epstein and Zin (1989) preferences and reconciles the equity and risk-free rate puzzle with a low risk aversion coefficient. Leippold, Trojani, and Vanini (2008) extend the model of Veronesi (2000) and reconcile the excess volatility puzzle with a 
high equity premium and a low risk-free rate. Both papers use the robust control approach of Hansen and Sargent (2007) to describe aversion to ambiguity. The main difference in this paper is that the excess sensitivity of prices to news results from a discontinuity in the equilibrium signal-to-price map which occurs even in a simple static model.

This paper is also related to Garlappi, Uppal, and Wang (2007) and Kogan and Wang (2003). Garlappi, Uppal, and Wang (2007) discuss the effect of ambiguity on mean/variance portfolio choice and Kogan and Wang (2003) discuss the implications of ambiguity for the cross sectional properties of asset returns. In both papers investors have perfect knowledge about the covariance matrix but are ambiguous about the mean return vector of asset returns. The main difference of this paper is that I focus on how ambiguity about the informativeness of news and hence imperfect knowledge of the posterior mean and variance of fundamentals affects optimal portfolios of investors and, more importantly, equilibrium prices.

Other papers that discuss ambiguity aversion are summarized below. Epstein and Schneider (2007) describe how ambiguity averse investors can learn and show with a simple portfolio choice problem how this can lead to more stock market participation. Chen and Epstein (2002) study the implications of ambiguity aversion on equilibrium prices in a representative agent continuous time exchange economy. A similar approach to allow for model uncertainty is considered by Hansen and Sargent (2007) (and coauthors) who apply robust control theory to economic problems. They also specify a set of models (or priors) to incorporate model uncertainty but an investor's uncertainty aversion is introduced through a function that penalizes models that differ from a prespecified reference model.

\section{Ambiguous Information}

In this section I show how investors who are averse to risk and ambiguity behave when the receive ambiguous information about the fundamentals of an asset. I adopt the model of Epstein and Schneider (2008) in which an investor considers multiple models that link information to fundamentals and then makes decisions with respect to the model that leads to the lowest expected utility. This behavior exhibits aversion to ambiguity (Knightian uncertainty) and is axiomatized by Gilboa and Schmeidler (1989) in a static setting and 
generalized to a dynamic setting by Epstein and Schneider (2003) 5

Suppose an investor receives a signal $\tilde{s}$ about the dividend $\tilde{d}$. A model consists of a marginal distribution of the dividend $\tilde{d}$ and a conditional distribution of the signal $\tilde{s}$ given the dividend $\tilde{d}$. The investor knows the marginal distribution of the dividend but lacks data and/or experience to know how to link this signal to the dividend and therefore doesn't know the conditional distribution of the signal given the dividend. The investor is averse to ambiguity and hence behaves as if she would have a set of models (a marginal and a set of conditionals) in mind when evaluating the outcome of a decisions.

Let $u(\cdot)$ denote the utility function of the investor, $m$ a model, $\mathcal{M}$ the set of all models considered by the investor, and $\mathrm{E}_{m}[\cdot]$ the expectation with respect to the belief generated by the model $m$. An ambiguity averse investor in the sense of Gilboa and Schmeidler (1989) chooses a portfolio $\theta$ to maximize

$$
\begin{aligned}
& \quad \inf _{m \in \mathcal{M}} \mathrm{E}_{m}[u(\tilde{w}) \mid \tilde{s}=s] \\
& \text { s.t. } \quad \tilde{w}=w_{0}+(\tilde{d}-p) \theta,
\end{aligned}
$$

in which $w_{0}$ denotes an investor's initial and $\tilde{w}$ her future wealth. The price of the risky asset is denoted by $p$ and the risk-free rate is normalized to zero.

This paper focuses on the effects of risk and ambiguity on asset prices and therefore it is important to emphasize the difference between aversion to risk and ambiguity. Specifically, the curvature of the utility function $u(\cdot)$ determines an investor's risk aversion whereas the size of $\mathcal{M}$ and the "inf" operator describe an investors ambiguity and aversion to ambiguity. If $\mathcal{M}$ is a singleton, then the investor is a standard expected utility maximizer in the sense of Savage (1954) and hence neutral to ambiguity. I use the terms ambiguity or aversion to ambiguity interchangeably because the axioms presented by Gilboa and Schmeidler (1989) do not allow to identify them separately 6

Suppose that both the marginal distribution of $\tilde{d}$ and the conditional distribution of $\tilde{s}$

\footnotetext{
${ }^{5}$ Other preferences that exhibit aversion to ambiguity are axiomatized in Klibanoff, Marinacci, and Mukerji (2005) and Maccheroni, Marinacci, and Rustichini (2006).

${ }^{6}$ This fact is emphasized in Routledge and Zin (2001). Moreover, Klibanoff, Marinacci, and Mukerji (2005) present a model of preferences that allows for a distinction between ambiguity and attitude towards ambiguity. The Gilboa and Schmeidler (1989) specification arises as a limiting case when investors have infinite aversion to ambiguity.
} 
given $\tilde{d}$ is normal. Specifically, there is a single normal marginal of $\tilde{d}$ :

$$
\tilde{d} \sim \mathrm{N}\left(\bar{d}, \sigma_{d}^{2}\right)
$$

and there is a family of conditional distributions of $\tilde{s}$ given $\tilde{d}$ :

$$
\tilde{s}=\tilde{d}+\tilde{\varepsilon}, \quad \tilde{\varepsilon} \sim \mathrm{N}\left(0, \sigma^{2}\right),
$$

in which $\sigma^{2} \in\left[\sigma_{a}^{2}, \sigma_{b}^{2}\right] \subseteq[0, \infty] 7$

Each model $m \in \mathcal{M}$ determines a conditional belief for $\tilde{d}$ given $\tilde{s}$ and hence standard normal-normal updating for each $\sigma^{2} \in\left[\sigma_{a}^{2}, \sigma_{b}^{2}\right]$ leads to

$$
\tilde{d} \mid \tilde{s}=s \sim \mathrm{N}_{\beta}\left(\bar{d}+\beta(s-\bar{d}), \sigma_{d}^{2}(1-\beta)\right), \quad \beta=\frac{\sigma_{d}^{2}}{\sigma_{d}^{2}+\sigma^{2}}
$$

It is convenient to describe the informativeness of the signal by beta and hence the set of conditional beliefs is given by $\left[\beta_{a}, \beta_{b}\right] \subseteq[0,1]$ with

$$
\begin{aligned}
& \beta_{a}=\sigma_{d}^{2} /\left(\sigma_{d}^{2}+\sigma_{b}^{2}\right) \\
& \beta_{b}=\sigma_{d}^{2} /\left(\sigma_{d}^{2}+\sigma_{a}^{2}\right) .
\end{aligned}
$$

The utility of an investor who is averse to ambiguous information and holds $\theta$ shares of the risky asset is therefore 8

$$
\min _{\beta \in\left[\beta_{a}, \beta_{b}\right]} \mathrm{E}_{\beta}\left[u\left(w_{0}+(\tilde{d}-p) \theta\right) \mid \tilde{s}=s\right] .
$$

Investors are more averse to ambiguous information if the set of models and hence the interval $\left[\beta_{a}, \beta_{b}\right]$ is large and therefore the degree of aversion to ambiguous information can be measured by $\beta_{b}-\beta_{a}$.

Suppose the investor has CARA utility over future wealth $\tilde{w}$; i.e. $u(\tilde{w})=-e^{-\gamma \tilde{w}}$. Then, the investor chooses $\theta$ to maximize her certainty equivalent 9 The certainty equivalent of

\footnotetext{
${ }^{7}$ I do not rule out the case $\left[\sigma_{a}^{2}, \sigma_{b}^{2}\right]=[0, \infty]$.

${ }^{8}$ The objective function is continuous and the feasible set is compact and hence I can replace the infimum by the minimum.

${ }^{9}$ The utility function is strictly increasing and hence optimizing $u(\mathrm{CE}(\cdot))$ is equivalent to optimizing $\mathrm{CE}(\cdot)$.
} 
the ambiguity averse investor with wealth $w_{0}$ is denoted by $\operatorname{CE}\left(\theta ; w_{0}, p, s\right)$. It is equal to the "worst case scenario certainty equivalent" of a Savage investor. Specifically,

$$
\mathrm{CE}\left(\theta ; w_{0}, p, s\right)=\min _{\beta \in\left[\beta_{a}, \beta_{b}\right]} \mathrm{CE}^{S}\left(\theta, \beta ; w_{0}, p, s\right)
$$

in which

$$
\mathrm{CE}^{S}\left(\theta, \beta ; w_{0}, p, s\right)=w_{0}+\left(\mathrm{E}_{\beta}[\tilde{d} \mid \tilde{s}=s]-p\right) \theta-\frac{1}{2} \gamma \operatorname{Var}_{\beta}[\tilde{d} \mid \tilde{s}=s] \theta^{2}
$$

denotes the certainty equivalent of a standard expected utility maximizer with wealth $w_{0}$ and subjective belief $\beta$ (a Savage investor with wealth $w_{0}$ and belief $\beta$ ).

The assumption of CARA-utility and normally distributed beliefs leads to a meanvariance portfolio choice problem in which the beta (informativeness) of the signal depends on the portfolio $\theta$ and the realization of the signal $s$. Specifically,

$$
\beta^{*}=\beta^{*}(\theta, s) \in \underset{\beta \in\left[\beta_{a}, \beta_{b}\right]}{\operatorname{argmin}} \operatorname{CE}^{S}\left(\theta, \beta ; w_{0}, p, s\right) .
$$

I discuss the "worst case scenario" for the mean and variance of the risky asset before I determine the certainty equivalent of the ambiguity averse investor (the "worst case scenario" certainty equivalent of a Savage investor).

Suppose the investor is long the asset $(\theta>0)$. Then the worst case scenario for the mean is a high beta signal if bad news arrives and a low beta signal if good news arrives because the mean is significantly adjusted downwards with bad news and moderately adjusted upwards with good news. Specifically,

$$
\min _{\beta \in\left[\beta_{a}, \beta_{b}\right]} \mathrm{E}_{\beta}[\tilde{d} \mid \tilde{s}=s]=\left\{\begin{array}{llc}
\bar{d}+\beta_{a}(s-\bar{d}) & \text { if } & s-\bar{d}>0 \\
\bar{d}+\beta_{b}(s-\bar{d}) & \text { if } & s-\bar{d}<0 \\
\bar{d} & \text { if } & s-\bar{d}=0 .
\end{array}\right.
$$

Similarly, if the investor is short the asset $(\theta<0)$, then the worst case scenario for the mean is a low beta signal when bad news arrives and a high beta signal when good news arrives.

On the other hand, the worst case scenario for the residual variance is always a low 
beta signal. Specifically,

$$
\max _{\beta \in\left[\beta_{a}, \beta_{b}\right]} \operatorname{Var}_{\beta}[\tilde{d} \mid \tilde{s}=s]=\sigma_{d}^{2}\left(1-\beta_{a}\right)
$$

The worst case scenarios for the mean and the variance can not be chosen independently of each other but depend on the beta of the signal and hence there is a tradeoff between the effects of beta on the mean and the variance when minimizing $C E^{S}(\beta ; \cdot)$.

The certainty equivalent of an ambiguity averse investor and its properties are determined in the next proposition.

Proposition 1. Let $\hat{\theta} \equiv-2(s-\bar{d}) /\left(\gamma \sigma_{d}^{2}\right)$. Then,

$$
C E\left(\theta ; w_{0}, p, s\right)= \begin{cases}C E^{S}\left(\theta, \beta_{a} ; w_{0}, p, s\right) & \text { if } \theta \leq \min (\hat{\theta}, 0) \\ C E^{S}\left(\theta, \beta_{b} ; w_{0}, p, s\right) & \text { if } \min (\hat{\theta}, 0)<\theta<\max (\hat{\theta}, 0) \\ C E^{S}\left(\theta, \beta_{a} ; w_{0}, p, s\right) & \text { if } \theta \geq \max (\hat{\theta}, 0)\end{cases}
$$

The certainty equivalent $C E(\cdot)$ is a continuous and concave function of the stock demand $\theta$. Moreover, it is continuously differentiable except for the critical values $\theta=0$ and $\theta=\hat{\theta}$ if $s \neq \bar{d}$.

Proof. See Appendix B.

The function $\mathrm{CE}^{S}(\cdot)$ does not depend on beta at the critical points 0 and $\hat{\theta}$ but is otherwise a linear function of beta because the conditional mean and the residual variance are linear in beta. Hence, the certainty equivalent of an ambiguity averse investor is either $\mathrm{CE}^{S}\left(\beta_{a} ; \cdot\right)$ or $\mathrm{CE}^{S}\left(\beta_{b} ; \cdot\right)$ and switches from one to the other at 0 and $\hat{\theta}$.

Figure 1 shows the certainty equivalent of three different Savage investors and the ambiguity averse investor as a function of the portfolio demand $\theta$. Specifically, the blue solid line shows the certainty equivalent of a Savage investor with belief $\beta=\beta_{a}$, the black dashed line shows the certainty equivalent of a Savage investor with belief $\beta=\left(\beta_{a}+\beta_{b}\right) / 2$, the red chain-dotted line shows the certainty equivalent of a Savage investor with belief $\beta=\beta_{b}$, and the black solid line shows the certainty equivalent of the ambiguity averse investor with belief $\beta=\beta^{*}$. The right graph shows the case when investors receive bad 
news $(s<\bar{d})$ and the left graph shows the case when investors receive good news $(s>\bar{d})$.
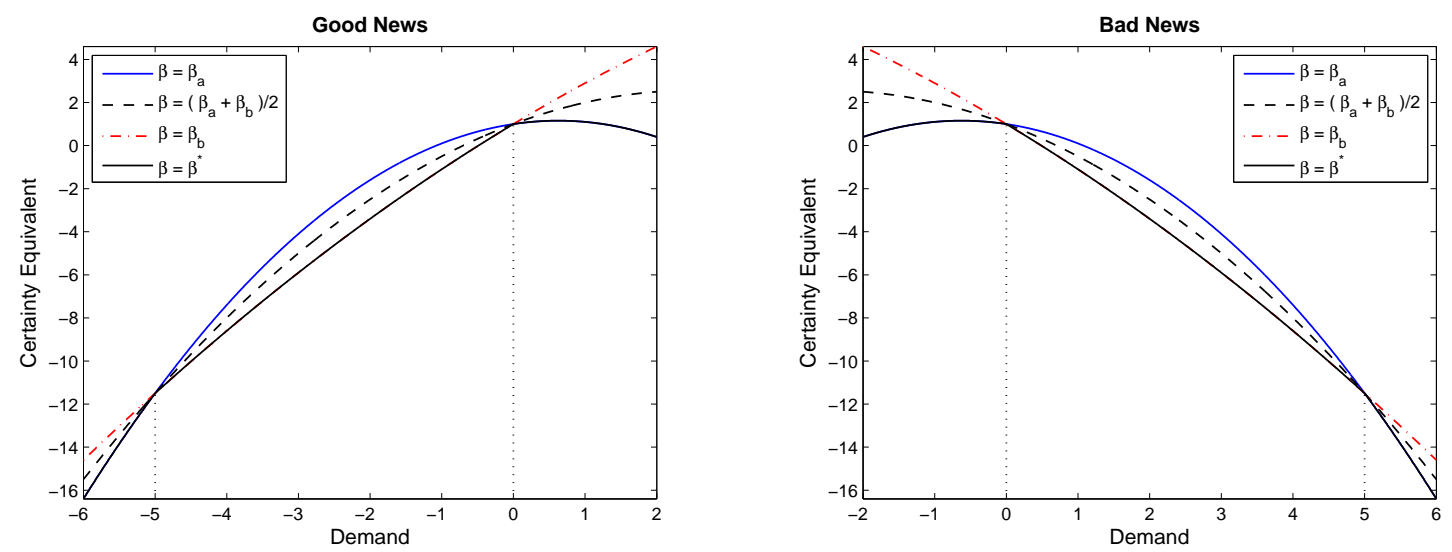

Figure 1: Expected Utility

Both figures show the certainty equivalent as a function of the demand $\theta$ when $\beta=\beta_{a}$ (blue solid line), $\beta=\left(\beta_{a}+\beta_{b}\right) / 2$ (black dashed line), $\beta=\beta_{b}$ (red chain-dotted line), and $\beta=\beta^{*}$ (black solid line). The parameters are $\bar{d}=5, \sigma_{d}=1, \gamma=1, w_{0}=1, p=5, \beta_{a}=1 / 5$, and $\beta_{b}=4 / 5$.

Suppose an investor receives bad news (right graph). If she is short the asset, then $\mathrm{CE}^{S}(\cdot)$ is uniquely minimized at $\beta_{a}$ because the worst case scenario for the mean and the residual variance is a low beta signal. On the other hand, if she is long the asset, then the worst case scenario for the mean is a high beta signal whereas the worst case scenario for the residual variance is a low beta signal. If the long position is sufficiently large $(\theta>\hat{\theta})$, then the variance dominates and $\mathrm{CE}^{S}(\cdot)$ is uniquely minimized at $\beta_{a} \cdot \mathrm{CE}^{S}(\cdot)$ is minimized at $\beta_{b}$ for small long positions in the asset $(\theta<\hat{\theta})$ because in this case the mean dominates. The long position for which the effects on the mean and the residual variance offset each other is $\hat{\theta} 10$ Similar arguments lead to the worst case scenario for an investor who receives good news (left graph).

If the signal confirms the expected value of the dividend $(s=\bar{d})$, then there is no ambiguity about the conditional mean and hence $\mathrm{CE}^{S}(\cdot)$ is uniquely minimized at $\beta_{a}$ for all portfolio positions because the worst case scenario for the residual variance is always a low beta signal. In other words, there is no kink in expected utility if $s=\bar{d}$.

To summarize, an investor who has CARA utility and is averse to ambiguous information in the sense of Gilboa and Schmeidler (1989) will evaluate the outcome of a portfolio

\footnotetext{
${ }^{10}$ If $\theta=0$, then there is no ambiguity and hence expected utility does not depend on beta.
} 
with respect the belief $\beta$ that leads to the lowest expected utility. Hence, the indifference curves of an investor who is either long or short in the risky asset have two kinks if the signal doesn't confirm the unconditional mean of the dividend and are otherwise smooth. The equilibrium price of the market portfolio when investors are averse to risk and ambiguous information is determined in the next section.

\section{Equilibrium}

In this section I derive the equilibrium price of the market portfolio when a representative investor receives an ambiguous signal about the fundamentals of the economy. I show that small changes in information about the value of the market portfolio can lead to drastic changes in the price of the market portfolio and better news do not always lead to a higher price. The proof of the existence of a representative investor is deferred to the next section.

Consider a discrete time economy with two dates 0 and 1 . There is a competitive market in a risk-free asset and a stock. The risk-free asset is in zero-net-supply and the stock is in positive supply normalized to one. The stock is a claim on a normally distributed liquidating dividend $\tilde{d}$ at date 1; i.e. $\tilde{d} \sim N\left(\bar{d}, \sigma_{d}^{2}\right)$. There is no ex ante ambiguity about the distribution of the dividend; i.e. $\bar{d}$ and $\sigma_{d}$ are known.

Suppose there is a representative investor with CARA-utility; i.e $u(x)=-e^{-\gamma x}$. There is no consumption at date zero. At date one the dividend $\tilde{d}$ is revealed and consumed by the representative investor. The risk-free asset is used as numeraire, so the risk-free rate is zero.

At date zero the investor receives an ambiguous signal about the dividend. The ambiguous signal is described by a family of conditionals. Specifically, $\tilde{s}=\tilde{d}+\tilde{\varepsilon}$ with $\tilde{\varepsilon} \sim \mathrm{N}\left(0, \sigma^{2}\right)$ and $\sigma^{2} \in\left[\sigma_{a}^{2}, \sigma_{b}^{2}\right]$. Ambiguity about the informativeness of the signal leads to the family of conditional beliefs for $\tilde{d}$ given $\tilde{s}$ described in equation (3).

The representative investor observes the realization of the ambiguous signal and chooses 
a portfolio $\theta$ to maximize

$$
\begin{aligned}
& \min _{\beta \in\left[\beta_{a}, \beta_{b}\right]} \mathrm{E}_{\beta}[u(\tilde{w}) \mid \tilde{s}=s] \\
& \text { s.t. } \quad \tilde{w}=w_{0}+(\tilde{d}-p) \theta,
\end{aligned}
$$

in which $\beta$ denotes the informativeness of the signal defined in equation (3) and $\beta_{a}$ and $\beta_{b}$ are defined in equations (44) and (5), respectively.

In equilibrium the representative investor holds the asset and consumes the liquidating dividend. Hence, the price of the asset at date one equals the liquidating dividend and $\theta=1$. The price at date zero depends on the signal and is determined below.

The equilibrium when the representative investor has standard expected utility preferences (in the sense of Savage (1954)) is provided in the next proposition. The proof is straightforward and thus omitted.

Proposition 2 (Savage Benchmark). If the representative investor is standard expected utility maximizers with subjective belief $\beta$, then

$$
p(s)=\mathrm{E}_{\beta}[\tilde{d} \mid \tilde{s}=s]-\gamma \operatorname{Var}_{\beta}[\tilde{d} \mid \tilde{s}=s] .
$$

The price is a strictly increasing continuous function of the signal because the conditional mean is strictly increasing and continuous in the signal and the conditional variance does not depend on the signal 11 This is no longer true when investors are averse to ambiguous information as the next theorem shows.

Theorem 1. Let $\hat{s}=\bar{d}-\gamma \sigma_{d}^{2} / 2$. There is a unique equilibrium stock price correspondence. Specifically,

$$
p(s) \in \begin{cases}\left\{\mathrm{E}_{\beta_{a}}[\tilde{d} \mid \tilde{s}=s]-\gamma \operatorname{Var}_{\beta_{a}}[\tilde{d} \mid \tilde{s}=s]\right\} & \text { if } s>\hat{s} \\ \mathcal{P}(\hat{s}) & \text { if } s=\hat{s} \\ \left\{\mathrm{E}_{\beta_{b}}[\tilde{d} \mid \tilde{s}=s]-\gamma \operatorname{Var}_{\beta_{b}}[\tilde{d} \mid \tilde{s}=s]\right\} & \text { if } s<\hat{s} .\end{cases}
$$

\footnotetext{
${ }^{11}$ The conditional expectation and variance in equation (14) are provided in Lemma 8 of Appendix [F
} 
Specifically, $p \in \mathcal{P}(\hat{s})$, if $\exists \beta \in\left[\beta_{a}, \beta_{b}\right]$ such that

$$
p=\mathrm{E}_{\beta}[\tilde{d} \mid \tilde{s}=\hat{s}]-\gamma \operatorname{Var}_{\beta}[\tilde{d} \mid \tilde{s}=\hat{s}]
$$

Proof. See Appendix D.

A brief description of the proof is as follows. If $s>\hat{s}(s<\hat{s})$, then by Proposition 1 there exists an open neighborhood of the market clearing stock demand $\theta=1$ for which $\mathrm{CE}^{S}(\cdot)$ is uniquely minimized at $\beta_{a}\left(\beta_{b}\right)$. Hence, the unique maximum of the certainty equivalent $\operatorname{CE}(\cdot)$ on this open neighborhood is attained at $\theta=1$, if and only if the price is equal to the Savage benchmark price, given in equation (14), when the mean and the variance are determined with respect to the conditional belief characterized by $\beta_{a}\left(\beta_{b}\right)$. Moreover, concavity of the certainty equivalent (see Proposition 1) implies that the local maximum is also a global maximum.

If $s=\hat{s}$, then there is an interval of equilibrium prices. Intuitively, if the signal $s$ attains the critical value $\hat{s}$, then $\hat{\theta}=1$ and hence by Proposition 1 the certainty equivalent is not differentiable at the market clearing demand $\theta=1$. Loosely speaking the interval of prices in this case consists of all prices that are needed to set all marginal utilities (the subdifferential of the certainty equivalent) to zero at $\theta=112$ Put it differently, the demand function is constant equal to one for a range of prices at the critical signal value $\hat{s}$ and hence all these prices are equilibrium prices 13

Let $p^{+}(\hat{s})$ denote the limit when $s$ approaches $\hat{s}$ from the right and $p^{-}(\hat{s})$ denote the limit of $p(s)$ when $s$ approaches $\hat{s}$ from the left. Specifically,

$$
\begin{aligned}
& p^{+}(\hat{s}) \equiv \lim _{s \downarrow \hat{s}} p(s)=\mathrm{E}_{\beta_{a}}[\tilde{d} \mid \tilde{s}=\hat{s}]-\gamma \operatorname{Var}_{\beta_{a}}[\tilde{d} \mid \tilde{s}=\hat{s}] \\
& p^{-}(\hat{s}) \equiv \lim _{s \uparrow \hat{s}} p(s)=\mathrm{E}_{\beta_{b}}[\tilde{d} \mid \tilde{s}=\hat{s}]-\gamma \operatorname{Var}_{\beta_{b}}[\tilde{d} \mid \tilde{s}=\hat{s}] .
\end{aligned}
$$

It is straightforward to verify that $\mathcal{P}(\hat{s})=\left[p^{+}(\hat{s}), p^{-}(\hat{s})\right]$ and

$$
\Delta p(\hat{s})=p^{+}(\hat{s})-p^{-}(\hat{s})=-\left(\beta_{b}-\beta_{a}\right) \frac{\gamma}{2} \sigma_{d}^{2}<0 .
$$

\footnotetext{
${ }^{12}$ See Appendix $\mathrm{A}$ for a definition and discussion of the subdifferential of a function.

${ }^{13}$ The demand function is determined in Proposition 3.
} 
The price of the market portfolio is a non-monotone and discontinuous correspondence of the signal (the price correspondence is upper hemicontinuous but not lower hemicontinuous and hence not continuous) 14 Figure 2 shows the equilibrium signal-to-price map.

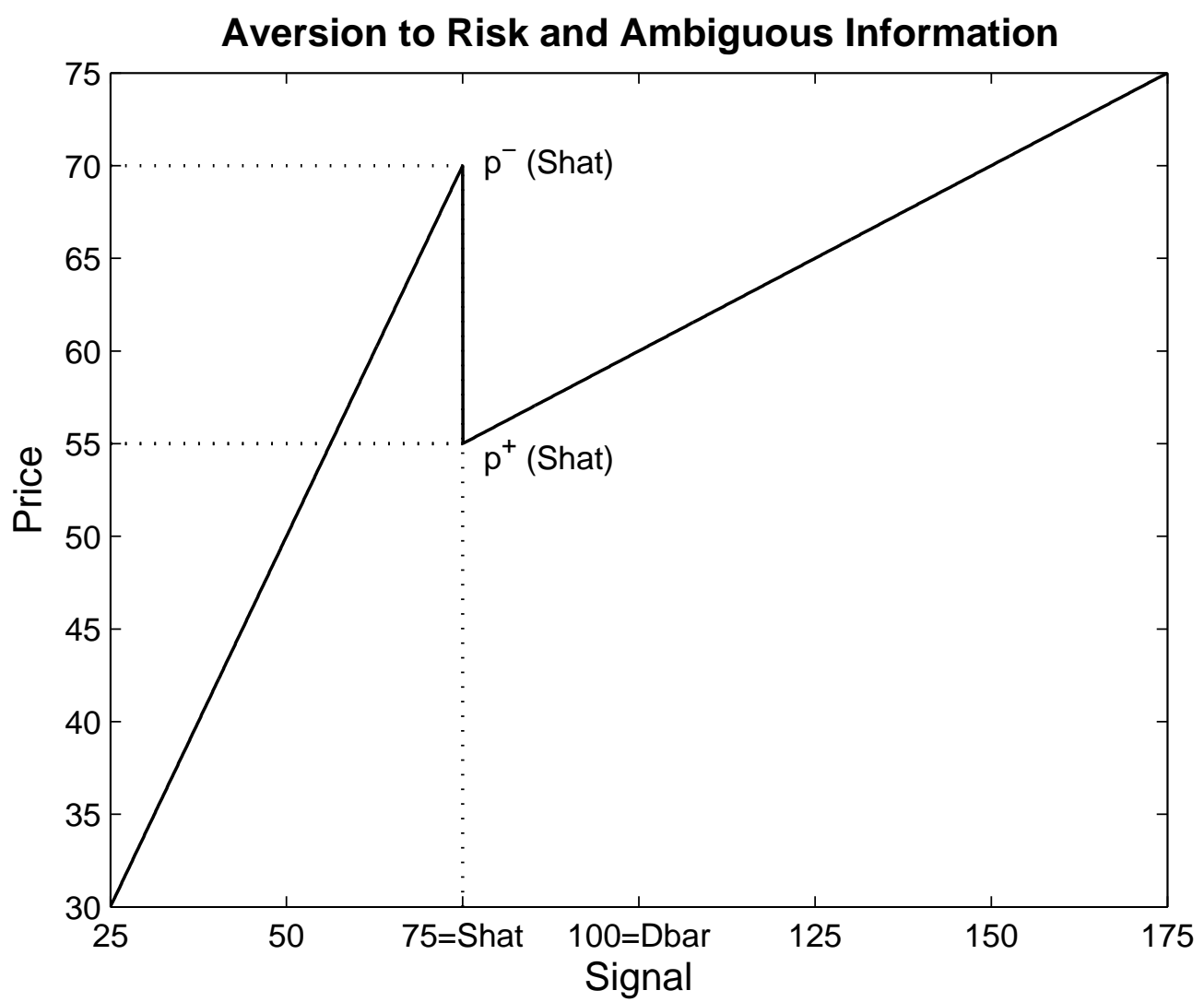

Figure 2: Equilibrium Signal-to-Price Map

This figure shows the equilibrium signal-to-price map of the market portfolio. The parameters are $\bar{d}=100, \sigma_{d}=5, \beta_{a}=1 / 5, \beta_{b}=4 / 5$, and $\gamma=2$. Moreover, $\hat{s}=75, p^{+}(\hat{s})=55$, and $p^{-}(\hat{s})=70$.

The price suddenly drops at the critical signal value $\hat{s}$ (which is equal to the unconditional mean minus half the unconditional risk premium of the asset) because the variance and hence the risk premium suddenly increases when the representative investor changes the interpretation of the information from a high beta signal to a low beta signal. Hence, small changes in news can lead to large changes in the price of the market portfolio.

\footnotetext{
${ }^{14}$ See Mas-Colell, Whinston, and Green (1995) Section M.H. for properties of correspondences.
} 
The critical signal value for which the representative investor switches the interpretation of the news is lower if the risk premium is large (the unconditional variance of the dividend and risk aversion are large) because in this case the news have to be really bad in order for the mean to dominate the risk premium.

There is no discontinuity in the equilibrium signal to price map if investors are standard expected utility maximizers $\left(\beta_{a}=\beta_{b}=\beta\right)$ and/or if they are risk neutral $(\gamma=0)$. Hence, it is possible to distinguish the effects of risk and ambiguity on the price of the market portfolio 15 Moreover, the price reacts more to bad news $(s<\hat{s})$ than to good news $(s>\hat{s})$ because the residual variance does not depend on the signal and the worst case scenario for the mean is a high beta signal for bad news and a low beta signal for good news 16

Figure 3 shows the equilibrium signal-to-price map for different aversion to risk and ambiguity. Specifically, the left graph shows the equilibrium signal-to-price map when $\gamma=0$ (black solid line), $\gamma=2$ (red chain-dotted line), and $\gamma=4$ (blue dashed line). The right graph shows it when $\left[\beta_{a}, \beta_{b}\right]=[0,1]$ (black solid line), $\left[\beta_{a}, \beta_{b}\right]=[1 / 10,1 / 2]$ (red chain-dotted line), and $\left[\beta_{a}, \beta_{b}\right]=[4 / 10,6 / 10]$ (blue dashed line).

\footnotetext{
${ }^{15}$ The issue of observational equivalence is often raised in the literature (see Backus, Routledge, and Zin (September 2004) page 37).

${ }^{16}$ Epstein and Schneider (2008) show that the price reacts more to bad news $(s<\bar{d})$ than to good news $(s>\bar{d})$ if investors are averse to ambiguous information but risk neutral.
} 

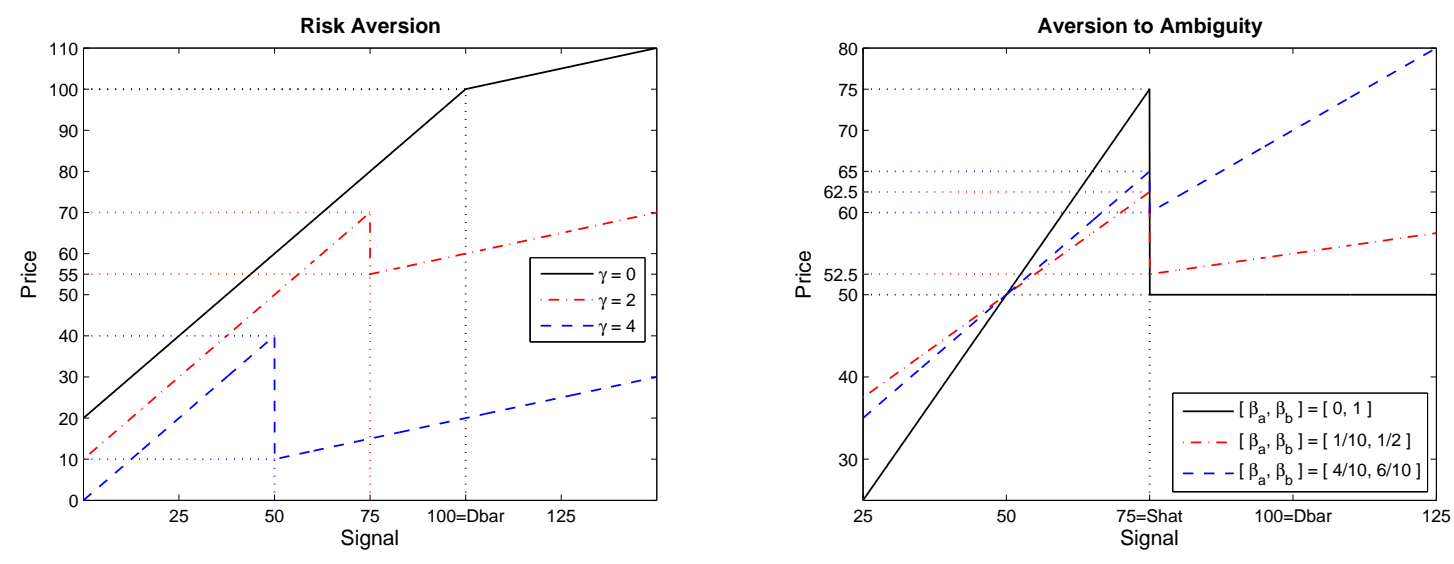

Figure 3: Different Aversion to Risk and Ambiguity

The left graph shows the equilibrium signal-to-price map when $\gamma=0$ (black solid line), $\gamma=2$ (red chain-dotted line), and $\gamma=4$ (blue dashed line). The parameters are: $\bar{d}=100$, $\sigma_{d}=5, \beta_{a}=1 / 5$, and $\beta_{b}=4 / 5$. The right figure shows the equilibrium signal-to-price map when $\left[\beta_{a}, \beta_{b}\right]=[0,1]$ (black solid line), $\left[\beta_{a}, \beta_{b}\right]=[1 / 10,1 / 2]$ (red chain-dotted line), and $\left[\beta_{a}, \beta_{b}\right]=[4 / 10,6 / 10]$ (blue dashed line). The parameters are: $\bar{d}=100, \sigma_{d}=5$, and $\gamma=2$.

If $\gamma=0$, then the price is continuously increasing in the signal $s$ with a kink at $s=\bar{d} 17$ This case is shown by the black solid line in the left graph of Figure 3. However, if $\gamma>0$, then the price is neither continuous nor monotone in the signal which is illustrated by the blue dashed line and the red chain-dotted line in the left graph of Figure 3 . The point $\hat{s}$ at which the representative investor switches the interpretation of the information moves to the left when risk aversion increases. Specifically, $\hat{s}=75$ if $\gamma=2$ and $\hat{s}=50$ if $\gamma=4$. Hence strong price reactions only occur for very bad news if risk aversion and hence the risk premium is large. Moreover, the size of the price drop increases with risk aversion because the the difference in the risk premium for low and high beta signals increases with risk aversion. The price drops by 15 when $\gamma=2$ and 30 when $\gamma=4$.

The right graph of Figure 3 shows that there is a large price reaction to bad news when

\footnotetext{
${ }^{17}$ The equilibrium stock price when investors are risk neutral and averse to ambiguous information simplifies to (see Epstein and Schneider (2008))

$$
\begin{aligned}
p(s) & =\min _{\beta \in\left[\beta_{a}, \beta_{b}\right]} \mathrm{E}_{\beta}[\tilde{d} \mid \tilde{s}=s] \\
& =\bar{d}+\beta_{a} \max (s-\bar{d}, 0)+\beta_{b} \min (s-\bar{d}, 0)
\end{aligned}
$$


$\beta_{b}$ is large and a moderate price reaction to good news when $\beta_{a}$ is low because the signal is treated as high beta $\left(\beta_{b}\right)$ for bad news and low beta $\left(\beta_{a}\right)$ for good news. The price drop is increasing in the aversion to ambiguous information measured by $\beta_{b}-\beta_{a}$ because the difference in the risk premium for low and high beta signals increases. Specifically, the price drops by 25 when $\beta_{b}-\beta_{a}=1$ (black solid line), by 10 when $\beta_{b}-\beta_{a}=3 / 8$ (red chain-dotted line), and by 5 when $\beta_{b}-\beta_{a}=1 / 5$ (blue dashed line).

\section{II.A Unconditional Moments of Price Changes}

In this section I discuss the effects of aversion to risk and ambiguity on the unconditional distribution of changes in the price of the market portfolio. Specifically, I compute the mean, variance, skewness, and excess kurtosis of the price change $\tilde{d}-p(\tilde{s})$. The "true" or "objective" conditional distribution (the distribution an econometrician would observe) of $\tilde{d}$ given $\tilde{s}$ is characterized by $\beta \in\left[\beta_{a}, \beta_{b}\right]$. The moments are plotted as a function of $\beta$ for three different cases: (i) the Savage case when $\gamma=1$ and the objective belief $\beta$ coincides with the subjective belief of the Savage investor, (ii) the Epstein-Schneider case when $\gamma=0$ and $\left[\beta_{a}, \beta_{b}\right]=[1 / 5,4 / 5]$, and (iii) the case when investors are averse to risk and ambiguity. The first case is represented by the blue dashed line in all four graphs of Figure 4, the second case is represented by the red chain-dotted line in all four graphs, and the last case is represented by the black solid line when $\gamma=1$ and $\left[\beta_{a}, \beta_{b}\right]=[1 / 5,4 / 5]$ in all four graphs and by the black dotted line when $\gamma=5$ and $\left[\beta_{a}, \beta_{b}\right]=[1 / 5,4 / 5]$ for all graphs except the top left.

If investors are averse to risk and ambiguity, then the expected price change is larger than in either the Savage or Epstein-Schneider case because investors require both a risk premium and an ambiguity premium to hold the market portfolio (see top left graph of Figure (4). Aversion to ambiguity leads to a larger variance than risk aversion because in the former investors can drastically change the interpretation of the information. This leads to a kink in the Epstein-Schneider case and a discontinuity when investors are averse to risk and ambiguity. The skewness and excess kurtosis in the Savage case is zero. Aversion to ambiguity tends to result in fatter tails while the skewness is positive for low risk aversion and negative for large risk aversion. Intuitively, the price change is positively skewed when the representative investor is risk neutral because the aggregate dividend is normally 
distributed and the price reacts more to bad signals than to good signals. If the investor is risk averse, then the discontinuity leads to a large variance of the price change conditional on negative news and hence, for sufficiently high risk aversion, to a negative skewness of price changes.
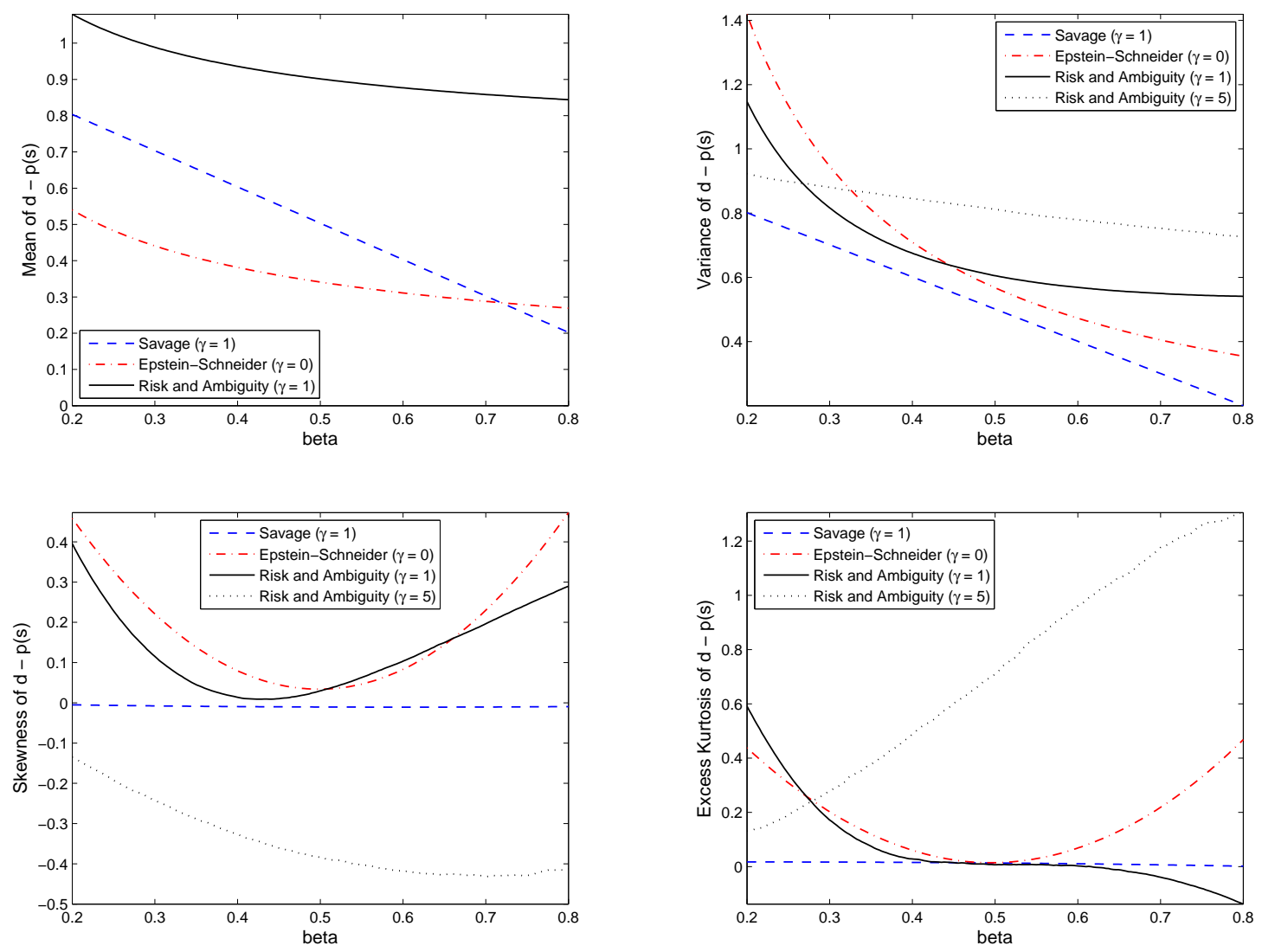

Figure 4: Unconditional Moments of Price Changes

The top left graph shows the mean, the top right graph shows the variance, the bottom left graph shows the skewness, and the bottom right graph shows the excess kurtosis of $\tilde{d}-p(\tilde{s})$ as a function of the objective distribution characterized by $\beta \in\left[\beta_{a}, \beta_{b}\right]$. In all four graphs the blue dashed line represents the Savage case when $\gamma=1$ and the subjective belief coincides with $\beta \in\left[\beta_{a}, \beta_{b}\right]$. The red chain dotted line represents the Epstein-Schneider case when $\gamma=0$ and $\left[\beta_{a}, \beta_{b}\right]=[1 / 5,4 / 5]$. The case when investors are averse to risk and ambiguity is shown by the black solid line when $\gamma=1$ and $\left[\beta_{a}, \beta_{b}\right]=[1 / 5,4 / 5]$ in all four graphs and by the black dotted line when $\left[\beta_{a}, \beta_{b}\right]=[1 / 5,4 / 5]$ and $\gamma=5$ four all graphs except the top left. The parameters are $\bar{d}=5$ and $\sigma_{d}=1$. 
Figure 5 shows the unconditional moments of the price change $\tilde{d}-p(\tilde{s})$ as a function of ambiguity $\beta_{b}-\beta_{a}$. The objective distribution is characterized by $\beta=\left(\beta_{a}+\beta_{b}\right) / 2$. Ambiguity $\beta_{b}-\beta_{a}$ increases from zero to one such that $\beta$ is always the midpoint of the interval $\left[\beta_{a}, \beta_{b}\right]$. In all four graphs the blue dashed line represents the Savage case when $\gamma=1$ and the subjective belief coincides with $\beta$. The red chain dotted line represents the Epstein-Schneider case when $\gamma=0$. The case when investors are averse to risk and ambiguity is shown by the black solid line when $\gamma=1$ in all four graphs and by the black dotted line when $\gamma=5$ four all graphs except the top left. The parameters are $\bar{d}=5$ and $\sigma_{d}=1$.

The mean and variance are strictly increasing in the degree of aversion to ambiguity. The rate of increase is linear for the mean and does not depend on risk aversion whereas the variance increases exponentially at a rate that is increasing in the level of risk aversion. The excess kurtosis is increasing in ambiguity for large levels of risk aversion but remains constant if risk aversion is close to zero. The skewness of price changes is increasing for low risk aversion and decreasing for large risk aversion. 

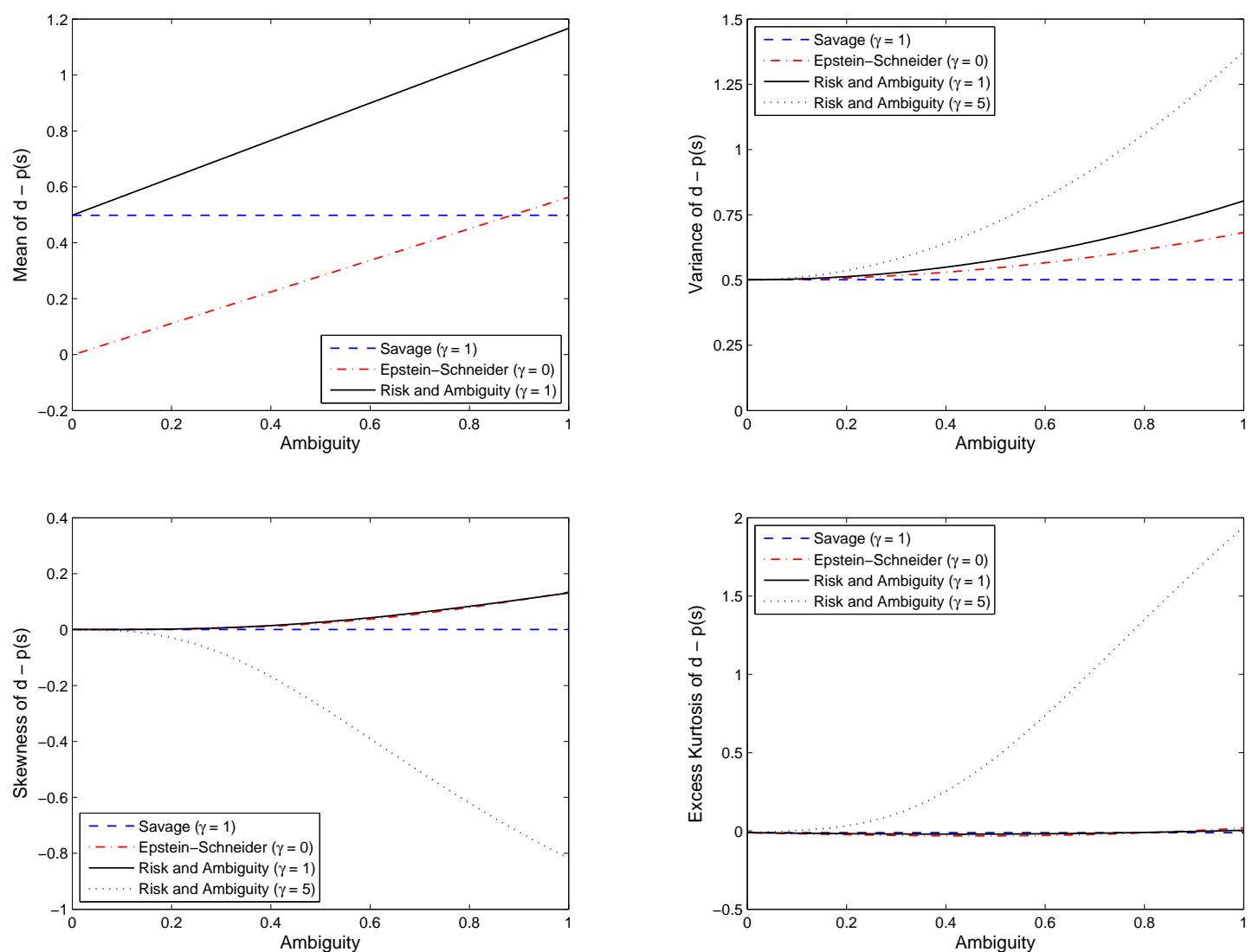

Figure 5: Effects of Ambiguity Aversion on Unconditional Moments of Price Changes

The top left graph shows the mean, the top right graph shows the variance, the bottom left graph shows the skewness, and the bottom right graph shows the excess kurtosis of $\tilde{d}-p(\tilde{s})$ as a function of ambiguity $\beta_{b}-\beta_{a}$. The objective distribution is characterized by $\beta=\left(\beta_{a}+\beta_{b}\right) / 2$. In all four graphs the blue dashed line represents the Savage case when $\gamma=1$ and the subjective belief coincides with $\beta$. The red chain dotted line represents the Epstein-Schneider case when $\gamma=0$. The case when investors are averse to risk and ambiguity is shown by the black solid line when $\gamma=1$ in all four graphs and by the black dotted line when $\gamma=5$ four all graphs except the top left. The parameters are $\bar{d}=5$ and $\sigma_{d}=1$. Ambiguity $\beta_{b}-\beta_{a}$ increases from zero to one such that $\beta$ is always the midpoint of the interval $\left[\beta_{a}, \beta_{b}\right]$. 


\section{Portfolio Choice}

In this section I determine the optimal portfolio of an investor who can invest in a risky asset and a risk-free asset and receives an ambiguous signal about the value of the risky asset. I show that (i) there is a range of prices for which investors do not change their short position in the risky asset when they receive good news, (ii) there is a range of prices for which investors do not change their long position in the risky asset when they receive bad news, and (iii) there is a range of prices for which investors are neither long or short in the risky asset when they receive good or bad news.

I have shown in Section \that an investor with CARA-utility and aversion to ambiguous information chooses a portfolio $\theta \in \mathcal{R}$ to maximize

$$
\mathrm{CE}\left(\theta ; w_{0}, p, s\right)=\min _{\beta \in\left[\beta_{a}, \beta_{b}\right]}\left\{w_{0}+\left(\mathrm{E}_{\beta}[\tilde{d} \mid \tilde{s}=s]-p\right) \theta-\frac{1}{2} \gamma \operatorname{Var}_{\beta}[\tilde{d} \mid \tilde{s}=s] \theta^{2}\right\} .
$$

The investor is a mean-variance optimizer and averse to ambiguous information and hence the mean-variance frontier depends on the realization and the informativeness of the signal. The solution to the portfolio choice problem in this case is provided in the next proposition.

Proposition 3. The optimal demand function for an investor with risk aversion $\gamma$ and aversion to ambiguous information described by $\left[\beta_{a}, \beta_{b}\right]$ is continuously decreasing if $s=\bar{d}$ and continuously non-increasing if $s \neq \bar{d}$. Specifically,

$$
\theta(p)= \begin{cases}\frac{\mathrm{E}_{\beta_{a}}[\tilde{d} \mid \tilde{s}=s]-p}{\gamma \operatorname{Var}_{\beta_{a}}[\tilde{d} \mid \tilde{s}=s]} & p \leq p_{1} \\ -\frac{2}{\gamma \sigma_{d}^{2}} \min (s-\bar{d}, 0) & p_{1}<p \leq p_{2} \\ \frac{\mathrm{E}_{\beta_{b}}[\tilde{d} \mid \tilde{s}=s]-p}{\gamma \operatorname{Var}_{\beta_{b}}[\tilde{d} \mid \tilde{s}=s]} & p_{2}<p \leq p_{3} \\ -\frac{2}{\gamma \sigma_{d}^{2}} \max (s-\bar{d}, 0) & p_{3}<p \leq p_{4} \\ \frac{\mathrm{E}_{\beta_{a}}[\tilde{d} \mid \tilde{s}=s]-p}{\gamma \operatorname{Var}_{\beta_{a}}[\tilde{d} \mid \tilde{s}=s]} & p>p_{4},\end{cases}
$$


in which

$$
\begin{aligned}
& p_{1}=\mathrm{E}_{\beta_{a}}[\tilde{d} \mid \tilde{s}=s]+\frac{2}{\sigma_{d}^{2}} \operatorname{Var}_{\beta_{a}}[\tilde{d} \mid \tilde{s}=s] \min (s-\bar{d}, 0) \\
& p_{2}=\mathrm{E}_{\beta_{b}}[\tilde{d} \mid \tilde{s}=s]+\frac{2}{\sigma_{d}^{2}} \operatorname{Var}_{\beta_{b}}[\tilde{d} \mid \tilde{s}=s] \min (s-\bar{d}, 0) \\
& p_{3}=\mathrm{E}_{\beta_{b}}[\tilde{d} \mid \tilde{s}=s]+\frac{2}{\sigma_{d}^{2}} \operatorname{Var}_{\beta_{b}}[\tilde{d} \mid \tilde{s}=s] \max (s-\bar{d}, 0) \\
& p_{4}=\mathrm{E}_{\beta_{a}}[\tilde{d} \mid \tilde{s}=s]+\frac{2}{\sigma_{d}^{2}} \operatorname{Var}_{\beta_{a}}[\tilde{d} \mid \tilde{s}=s] \max (s-\bar{d}, 0) .
\end{aligned}
$$

Proof. See Appendix C.

I use the subdifferential of the certainty equivalent which I define and calculate in the Appendix to prove Proposition 3. To provide some more intuition consider three different investors: a low beta Savage investor (a standard expected utility maximizer with subjective belief $\beta_{a}$ ), a high beta Savage investor (a standard expected utility maximizer with subjective belief $\beta_{b}$ ), and an investor with aversion to ambiguity described by $\left.\left[\beta_{a}, \beta_{b}\right]\right) 18$

Let $\theta_{a}(p)$ denote the optimal demand of a low beta Savage investor, $\theta_{b}(p)$ the optimal demand of a high beta Savage investor, and $\theta(p)$ the optimal demand of an ambiguity averse investor. Maximizing the certainty equivalent given in equation (8) evaluated at $\beta_{a}$ and $\beta_{b}$ leads to the optimal demand of the low beta and high beta Savage investor. Specifically,

$$
\begin{aligned}
\theta_{a}(p) & =\frac{\mathrm{E}_{\beta_{a}}[\tilde{d} \mid \tilde{s}=s]-p}{\gamma \operatorname{Var}_{\beta_{a}}[\tilde{d} \mid \tilde{s}=s]} \\
\theta_{b}(p) & =\frac{\mathrm{E}_{\beta_{b}}[\tilde{d} \mid \tilde{s}=s]-p}{\gamma \operatorname{Var}_{\beta_{b}}[\tilde{d} \mid \tilde{s}=s]}
\end{aligned}
$$

The proof is straightforward and thus omitted.

The optimal demand of the ambiguity investor is equal to the optimal demand of the low beta Savage investor when the signal confirms the expected value of the dividend $(s=\bar{d})$ because in this case there is only ambiguity about the residual variance and hence there is no kink in expected utility (see Proposition 1).

\footnotetext{
${ }^{18}$ The discussion follows closely Routledge and Zin (2001).
} 
If the price satisfies $p \leq p_{1}$ or $p \geq p_{4}$, then the ambiguity averse investor behaves like a low beta Savage investor and hence $\theta(p)=\theta_{a}(p)$. For instance, suppose an investor receives good news $(s=125>\bar{d}=100)$ and the price of the asset is high $(p=192.5)$. Then the certainty equivalent of the ambiguity averse investor coincides with the certainty equivalent of a low beta Savage investor for large short positions and all long positions in the asset, and coincides with the certainty equivalent of a high beta Savage investor for small long positions. In this case, the optimal demand of the ambiguity averse investor satisfies the first order condition of the low beta Savage investor because the price is so high $\left(p=192.5>p_{4}=147.5\right)$ such that the optimal short position $\left(\theta^{*}=-2\right)$ is larger than the critical value $\hat{\theta}=-1.19$ This case is illustrated in the left graph of Figure 6. Similarly, if investors receive bad news $(s=25<\bar{d}=100)$ and $p_{2} \leq p \leq p_{3}$, then the ambiguity averse investor behaves like a high beta Savage investor and hence $\theta(p)=\theta_{b}(p)$. This case is illustrated in the right graph of Figure 6.
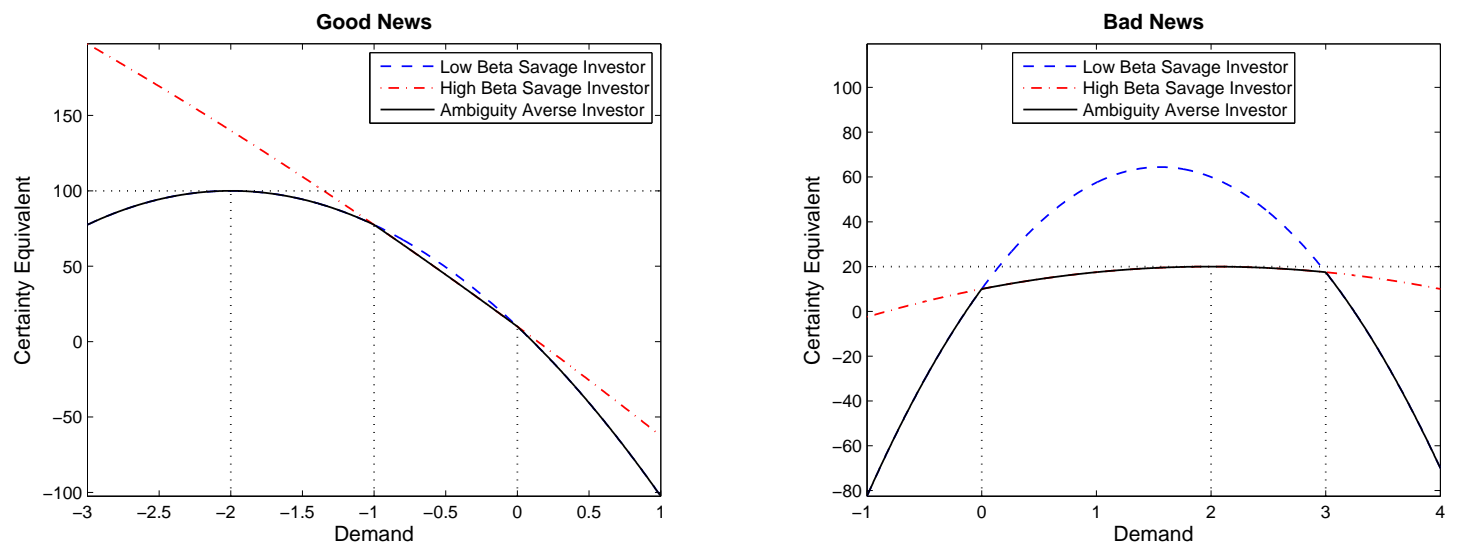

Figure 6: Expected Utility is Smooth at an Optimum

The blue dashed line shows the certainty equivalent of a low beta Savage investor, the red chain-dotted line shows the certainty equivalent of a high beta Savage investor, and the black solid line shows the certainty equivalent of an ambiguity averse investor. The left graph shows the certainty equivalent as a function of the demand when $s=125$ and $p=125$ whereas the right graphs shows the certainty equivalent when $s=25$ and $p=22.5$. The parameters are $w_{0}=10, \bar{d}=100, \sigma_{d}=5, \beta_{a}=1 / 10, \beta_{b}=9 / 10$, and $\gamma=2$.

However, if $p_{1}<p<p_{2}$ or $p_{3}<p<p_{4}$, then the behavior of an ambiguity averse investor is different than the behavior of a Savage investor. Specifically, a marginal change of the price in this range doesn't change the optimal demand. To illustrate that suppose the price of the risky asset is 43.75 and the realization of the signal is 62.5 . The ambiguity

\footnotetext{
${ }^{19}$ The subdifferential of the certainty equivalent is single-valued and equal to zero at the optimal demand.
} 
averse investor behaves like a high beta Savage investor and increases her demand until the critical value $\hat{\theta}=1.5$. If she would continue to increase her long position, then the utility would still increase and hence, $\theta=1.5$ would not be optimal for a high beta Savage investor. However, at $\theta=\hat{\theta}=1.5$ the ambiguity averse investor does no longer behave like a high beta Savage investor. Specifically, a further increase of the long position would mean a change to the behavior of a low beta Savage investor. However, for a low beta Savage investor the price of the risk asset is too large and thus her expected utility would go down with a further increase of the long position. Hence, to be at the kink is optimal for the ambiguity averse investor 20 This case is illustrated in the left graph of Figure 7.

The behavior of an ambiguity averse investor is distinctly different than the behavior of a Savage investor at $\hat{\theta}=1.5$. To see this suppose the price increases from $p<p_{2}$ to $p=p_{2}$. It is still optimal for the ambiguity averse investor to hold $\hat{\theta}=1.5$ shares of the risky asset even though expected utility has decreased from 40 to 17.5 because of the rise in the price of the risky asset. This case is illustrated in Figure 7 . Hence, a change in the price does not change the portfolio but it changes expected utility. In contrast, a Savage investor would reduces her long position when the price goes up 21

The investor doesn't hold the asset if its price lies between the highest and lowest valuation of the dividend given the signal . This is the well known non-participation result discussed in Dow and Werlang (1992), Cao, Wang, and Zhang (2005), and Epstein and Schneider (2007). However, if investors receive ambiguous information, then the investor neither changes her long nor her short position if the price lies between the highest and lowest valuation of the mean minus $\hat{\theta}$ times the risk premium of the asset. Hence, the demand function is flat for two price ranges because the worst case scenario belief depends on the portfolio and the signal.

Figure 8 shows the optimal demand function when $s>\bar{d}$ (red dashed line), $s=\bar{d}$ (black line), and $s<\bar{d}$ (blue chain-dotted line). Suppose the investor receives bad news $(s<\bar{d}$ and hence $\hat{\theta}>0$ ). Then there is a range of low prices for which she does not change her long position $\hat{\theta}$. Hence, the demand for the asset may be larger, than if she would have

\footnotetext{
${ }^{20}$ The subdifferential of the certainty equivalent is multi-valued and contains zero at the optimal demand.

${ }^{21}$ If the price of the risky asset lies between the highest and lowest valuation of the asset, then it is optimal not to hold the asset. In this case a marginal change in the price neither changes the position in the asset nor the utility value.
} 

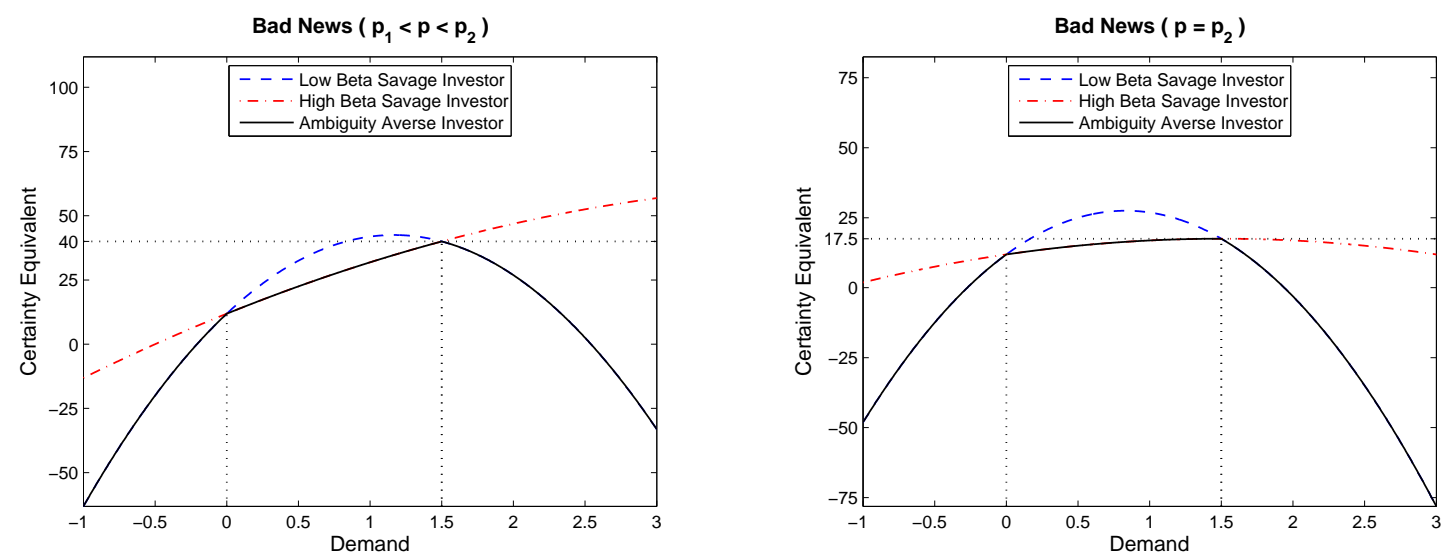

Figure 7: Expected Utility has a Kink at the Optimum

The blue dashed line shows the certainty equivalent of a low beta Savage investor, the red chain-dotted line shows the certainty equivalent of a high beta Savage investor, and the black solid line shows the certainty equivalent of an ambiguity averse investor as a function of demand. The left graph shows the certainty equivalent when $p=43.75$ and the right graph shows the certainty equivalent when $p=58.75$. The parameters are $\bar{d}=100, \sigma_{d}=5$, $\beta_{a}=1 / 10, \beta_{b}=9 / 10, \gamma=2, s=62.5$, and $w_{0}=11.875$.

received good news. Intuitively, the investor changes the interpretation of bad news for low prices but not for good news.

\section{Aggregation}

Consider the one period economy described in Section $\amalg$ and suppose there are $\mathrm{H}$ investors that all receive the same ambiguous signal but may differ with respect to initial wealth and aversion to risk and ambiguity. Let $w_{0 h}$ denote investor $h$ 's initial wealth, $\gamma_{h}$ her risk aversion coefficient, and $\left[\beta_{a h}, \beta_{b h}\right]$ the interval that describe her aversion to ambiguous information.

Definition 1 (Equilibrium). The signal to-price-map $p(s)$ is an equilibrium $\forall s \in \mathcal{R}$ if and only if (i) each investor chooses a portfolio $\theta_{h}$ to maximize

$$
\inf _{\beta_{h} \in\left[\beta_{a h}, \beta_{b h}\right]} \mathrm{E}_{\beta_{h}}\left[u_{h}\left(w_{0 h}+(\tilde{d}-p(s)) \theta_{h}\right) \mid \tilde{s}=s\right], \quad \forall s \in \mathcal{R}
$$

and (ii) markets clear; i.e. $\sum_{h=1}^{H} \theta_{h}=1$.

There exists a representative investor if all investors are standard expected utility max- 


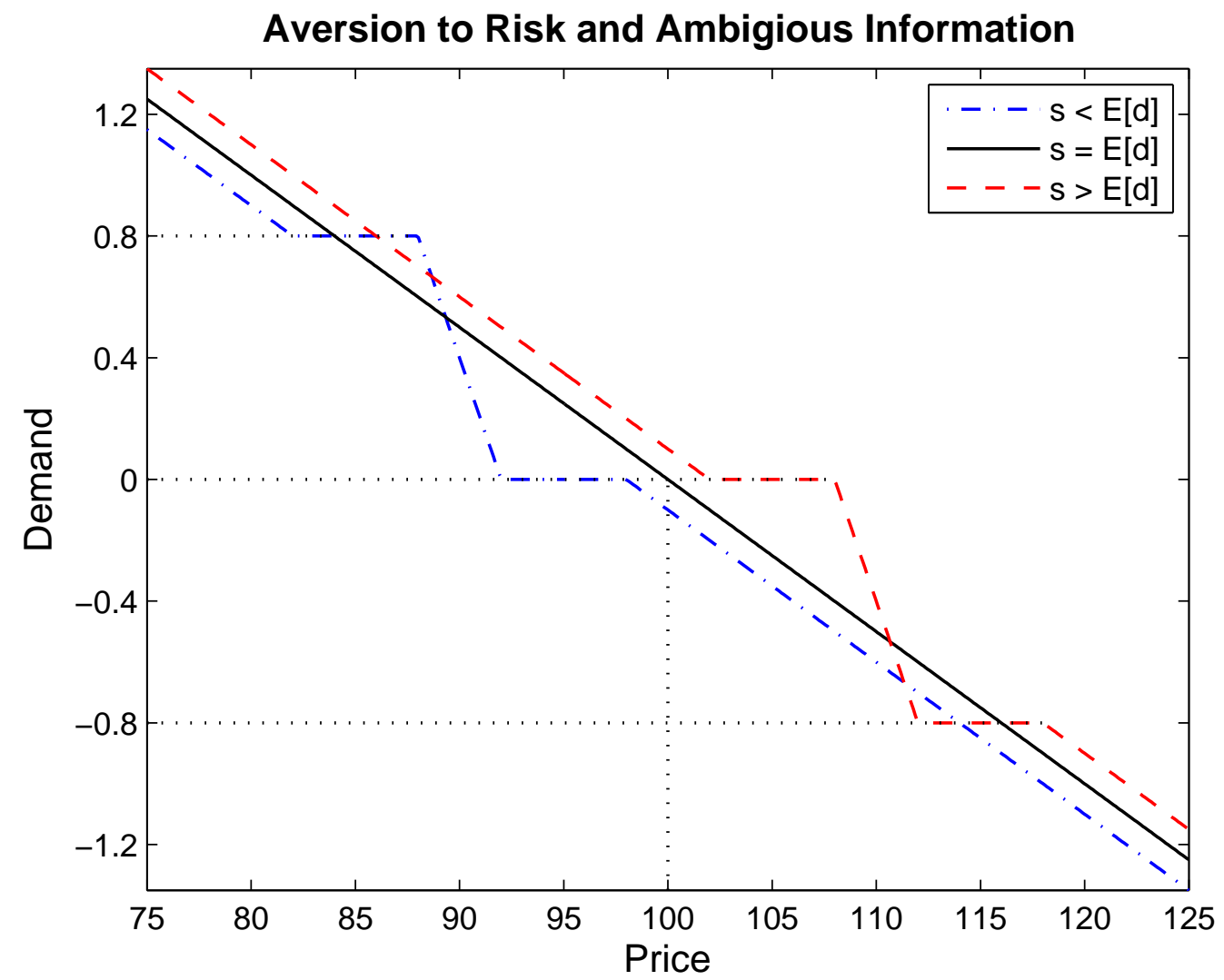

This figures shows optimal demand of an ambiguity averse investor with bad news $(s=90$, blue chain-dotted line), confirming news $(s=100$, black line), and good news $(s=110$, red dashed line). The parameters are $E[d]=\bar{d}=100, \sigma_{d}=5, \beta_{a}=1 / 5, \beta_{b}=4 / 5$, and $\gamma=1$.

Figure 8: Optimal Demand 
imizers. I show in the next proposition that this is still true when all investors have the same aversion to ambiguous information.

Proposition 4 (Representative Investor). Assume that all investors have the same aversion to ambiguous information described by $\left[\beta_{a}, \beta_{b}\right]$. Then, there exists a representative investor with aversion to ambiguous information described by $\left[\beta_{a}, \beta_{b}\right]$, initial wealth equal to $w_{0}=$ $\sum_{h=1}^{H} w_{0 h}$, and absolute risk aversion equal to

$$
\gamma=\frac{1}{\sum_{h=1}^{H} \frac{1}{\gamma_{h}}} .
$$

Proof. See Appendix D.

Intuitively, the range of prices at which the demand function given in equation (22) changes its slope does not depend on risk aversion and hence individual demands can be added up as in the standard expected utility case.

Hence, the equilibrium signal-to-price map is given in Theorem 1 with $\gamma=1 / \sum_{h=1}^{H}\left(1 / \gamma_{h}\right)$ and $\left[\beta_{a h}, \beta_{b h}\right]=\left[\beta_{a}, \beta_{b}\right] \forall h$.

Suppose there is also heterogeneity in aversion to ambiguous information and define $\left[\beta_{a}, \beta_{b}\right] \equiv \bigcap_{h=1}^{H}\left[\beta_{a h}, \beta_{b h}\right]$. I show in the next proposition that there is a still a discontinuity in the equilibrium signal-to-price map when investors are heterogeneous in their aversion to ambiguous information.

Proposition 5 (Aggregation). Suppose there exists an equilibrium. Then there is a discontinuity in the equilibrium signal-to-price map if $\beta_{a} \neq \beta_{b}$. The interval of equilibrium prices is given by $\mathcal{P}$. Specifically, $p \in \mathcal{P}$, if $\exists \beta \in\left[\beta_{a}, \beta_{b}\right]$ such that

$$
p=\mathrm{E}_{\beta}[\tilde{d} \mid \tilde{s}=\hat{s}]-\gamma \operatorname{Var}_{\beta}[\tilde{d} \mid \tilde{s}=\hat{s}]
$$

in which $\hat{s}=\bar{d}-\gamma \sigma_{d}^{2} / 2$ and $\gamma=1 / \sum_{h=1}^{H}\left(1 / \gamma_{h}\right)$.

Proof. See Appendix D.

Figure 9 provides the intuition for the result. It shows the demand function for three investors and aggregate demand. All three investors differ with respect to their aversion 
to risk and ambiguity. The first investor has the highest degree of aversion to risk and ambiguity $\left(\gamma_{1}=5\right.$ and $\left.\left[\beta_{a 1}, \beta_{b 1}\right]=[1 / 10,9 / 10]\right)$. Her demand (blue solid line) thus doesn't change for a wide range of prices and is only moderately decreasing otherwise. The second investor has the lowest degree of aversion to ambiguous information $\left(\gamma_{2}=5 / 4\right.$ and $\left.\left[\beta_{a 2}, \beta_{b 2}\right]=[1 / 4,3 / 4]\right)$ whereas the third investor has the lowest degree of risk aversion $\left(\gamma_{3}=1\right.$ and $\left.\left[\beta_{a 3}, \beta_{b 3}\right]=[1 / 4,4 / 5]\right)$. The investor with the lowest degree of aversion to ambiguity determines the range of prices for which aggregate demand (black solid line) is flat; i.e. $\left[\beta_{a}, \beta_{b}\right]=[1 / 4,3 / 4]$. The individual demand for the second investor (green chaindotted line) is constant equal to $2 / 5$ for the same range of prices whereas the demand for the third investor (red dashed line) is $1 / 2$ and the demand for the first investor (blue solid line) is $1 / 10$ for an even wider range of prices. Hence, aggregate demand sums up to the market clearing stock demand of one and the range of prices at which the investor with the smallest aversion to ambiguity doesn't change her demand comprises the interval of equilibrium prices.

It immediately follows from Proposition 5 that:

Remark 1. There is no discontinuity in the equilibrium signal-to-price map if there is an investor who is neutral to ambiguous information.

Risk averse investors with subjective expected utility engage in betting or speculative trade if and only if their beliefs differ. If agents are also ambiguity averse, then there is speculative trade if and only if investors don't have a belief in common; i.e. $\bigcap_{h=1}^{H}\left[\beta_{a h}, \beta_{b h}\right]=\emptyset 22$ Hence, if there is no speculative trade, then there will be a discontinuity in the equilibrium signal to price map except for the case in which investors have only one belief in common. Put it differently, there is no discontinuity in the equilibrium signal-to-price map if there is speculative trade.

Remark 2. Assume that there is no speculative trade. There is a discontinuity in the equilibrium signal-to-price map if and only if investors have more than one belief in common.

\footnotetext{
${ }^{22}$ This is shown by Billot, Chateuneuf, and Gilboa (2000) for Gilboa and Schmeidler (1989) preferences and extended to a broader class of preferences that exhibit aversion to ambiguity, including the convex Choquet model of Schmeidler (1989), the smooth second-order prior models of Klibanoff, Marinacci, and Mukerji (2005) and Nau (2006), the second-order expected utility model of Ergin and Gul (2004), the multiplier model of Hansen and Sargent (2001), and the variational preferences of Maccheroni, Marinacci, and Rustichini (2006) by Rigotti, Shannon, and Strzalecki (2008).
} 


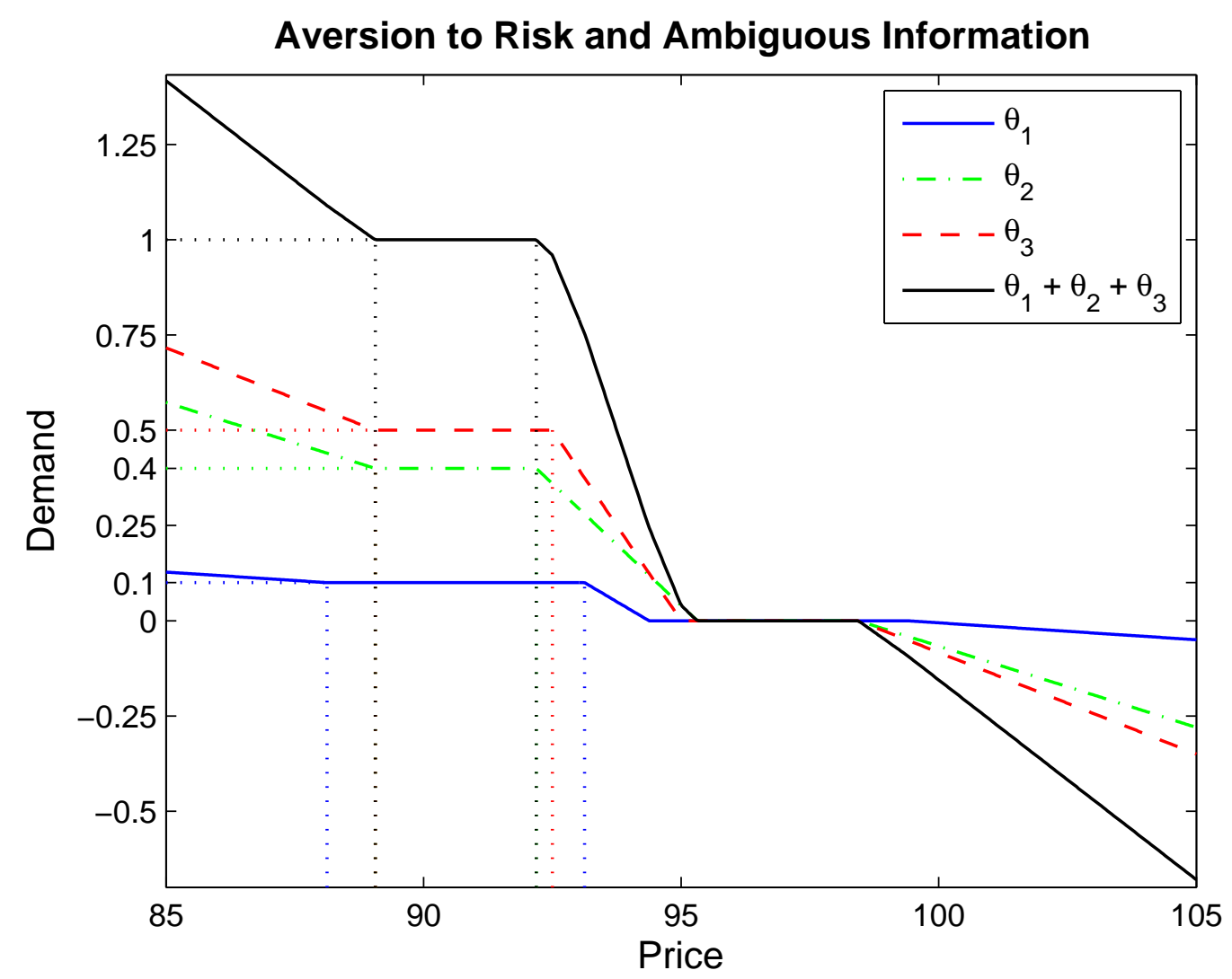

This figure shows the individual demand functions of three investors and aggregate demand. The investors differ with respect to their aversion to risk and ambiguity. Specifically, $\gamma_{1}=5$ and $\left[\beta_{a 1}, \beta_{b 1}\right]=[1 / 10,9 / 10], \gamma_{2}=5 / 4$ and $\left[\beta_{a 2}, \beta_{b 2}\right]=[1 / 4,3 / 4]$, and $\gamma_{3}=1$ and $\left[\beta_{a 3}, \beta_{b 3}\right]=$ $[1 / 4,4 / 5]$. The parameters are $\bar{d}=100, \sigma_{d}=5$, and $s=93.75$. The signal value $s$ is equal to the critical signal value $\hat{s}=93.75$ for an investor with risk aversion equal to $\gamma=1 / 2$ (aggregate risk aversion). The individual demand functions add up to one for a range of prices at which the second investor (an investor with aversion to ambiguity described by the intersection of all three investor's beliefs) doesn't change her demand. Hence, there is an interval of equilibrium stock prices.

Figure 9: Aggregation 


\section{Two Ambiguous Signals}

We know from Epstein and Schneider (2008) that investors who anticipate ambiguous information about the value of an asset require a premium to hold the asset. In this section I discuss the effects of aversion to risk and ambiguity on the value of the market portfolio when investor can learn from ambiguous signals about the underlying fundamentals. In this case a model that links this ambiguous information to fundamentals will affect the mean, the risk premium, and the ambiguity premium of the market portfolio. I show that there are two discontinuities in the equilibrium signal-to-price map. Specifically, there is a sudden drop in the equilibrium price of the market portfolio for bad news and a sudden increases in the equilibrium price for good news.

Consider a discrete time economy with four dates $0,1,2$, and 3 . There is a competitive market in a risk-free asset and a stock. The risk-free asset is in zero-net-supply and the stock is in positive supply normalized to one. The stock is a claim on a normally distributed liquidating dividend $\tilde{d}$ at date 3 ; i.e. $\tilde{d} \sim N\left(\bar{d}, \sigma_{d}^{2}\right)$. There is no ex ante ambiguity about the distribution of the dividend; i.e. $\bar{d}$ and $\sigma_{d}$ are known.

Suppose there is a representative investor with CARA-utility, i.e. $u(x)=-e^{-\gamma x}$ and $\gamma \geq 0$. There is no interim consumption. At date 3 the dividend $\tilde{d}$ is revealed and consumed by the representative investor. The risk-free asset is used as numeraire, so the risk-free rate is zero.

At date 2 the representative investor observes a noisy signal of the dividend. Specifically,

$$
\tilde{s}_{2}=\tilde{d}+\tilde{\varepsilon}_{2}, \quad \tilde{\varepsilon}_{2} \stackrel{\text { id }}{\sim} \mathrm{N}\left(0, \sigma_{2}^{2}\right)
$$

At date 1 the representative investor observes a noisy signal of $\tilde{s}_{2}$. Specifically,

$$
\tilde{s}_{1}=\tilde{s}_{2}+\tilde{\varepsilon}_{1}, \quad \tilde{\varepsilon}_{1} \stackrel{\mathrm{id}}{\sim} \mathrm{N}\left(0, \sigma_{1}^{2}\right)
$$

The representative investor is ambiguous about the informativeness of both signals and hence makes decision with respect to a family of signal noise distributions described by $\left[\sigma_{2 a}, \sigma_{2 b}\right]$ and $\left[\sigma_{1 a}, \sigma_{1 b}\right]$, respectively 23

\footnotetext{
${ }^{23}$ This simple two signal structure is a parsimonious way to study the impact of ambiguous information
} 
At date zero there is no ambiguity about the liquidating dividend but there is ambiguity about future information regarding the liquidating dividend described by the signals $\tilde{s}_{1}$ and $\tilde{s}_{2}$.

To summarize, a model or prior $m$ consists of a normal marginal distribution for fundamentals denoted by $\mu_{0}$ and two normal conditionals that link information to fundamentals. Specifically, the conditional distribution of $\tilde{s}_{1}$ given $s_{2}$ is denoted by $l_{1}$ and the conditional distribution of $\tilde{s}_{2}$ given $d$ is denoted by $l_{2}$. Hence, a model can be defined as a normal marginal and two normal conditionals; i.e. $m=\left(\mu_{0}, l_{1}, l_{2}\right)$. Let $\mathcal{M}$ denote the set of all possible models, $\mathcal{L}_{2}$ denote the set of all conditionals of $\tilde{s}_{2}$ given $d$ and $\mathcal{L}_{1}$ the set of all conditionals of $\tilde{s}_{1}$ given $s_{2}$. It follows that $\mathcal{M}=\left\{\mu_{0}\right\} \times \mathcal{L}_{1} \times \mathcal{L}_{2}$.

\section{Portfolio Choice}

For $t=0,1,2$, and 3 , let $w_{t}$ denote wealth at time $t, p_{t}$ the price of the stock at time $t$, and $\theta_{t}$ the number of shares of the stock in an investor's portfolio at time $t$. The budget constraint of an investor is

$$
w_{t+1}=w_{t}+\theta_{t}\left(p_{t+1}-p_{t}\right), \quad \forall t=0,1,2 .
$$

I use dynamic programming to solve for the optimal portfolio at each date 24 Specifically, an investor chooses $\theta_{2}$ to maximize

$$
\inf _{m \in \mathcal{M}} \mathrm{E}_{m}\left[u\left(w_{2}+\theta_{2}\left(\tilde{d}-p_{2}\right)\right) \mid \tilde{s}_{1}=s_{1}, \tilde{s}_{2}=s_{2}\right]
$$

The value function at date 2 is defined as

$$
V_{2}\left(w_{2}, s_{1}, s_{2}\right)=\sup _{\theta_{2} \in \mathcal{R}} \inf _{m \in \mathcal{M}} \mathrm{E}_{m}\left[u\left(w_{2}+\theta_{2}\left(\tilde{d}-p_{2}\right)\right) \mid \tilde{s}_{1}=s_{1}, \tilde{s}_{2}=s_{2}\right] .
$$

At date 1 an investor chooses $\theta_{1}$ to maximize

$$
\inf _{m \in \mathcal{M}} \mathrm{E}_{m}\left[V_{2}\left(w_{1}+\theta_{1}\left(p_{2}\left(\tilde{s}_{2}, s_{1}\right)-p_{1}\right), \tilde{s}_{2}, s_{1}\right) \mid \tilde{s}_{1}=s_{1}\right]
$$

on asset prices and changes in asset prices when investors are averse to risk and ambiguity. Moreover, it captures an investor's ability to learn over time about fundamentals from ambiguous information.

${ }^{24}$ Preferences are dynamically consistent (see Epstein and Schneider (2003)). 
The value function at date 1 is defined as

$$
V_{1}\left(w_{1}, s_{1}\right)=\sup _{\theta_{1} \in \mathcal{R}} \inf _{m \in \mathcal{M}} \mathrm{E}_{m}\left[V_{2}\left(w_{1}+\theta_{1}\left(p_{2}\left(\tilde{s}_{2}, s_{1}\right)-p_{1}\right), \tilde{s}_{2}, s_{1}\right) \mid \tilde{s}_{1}=s_{1}\right] .
$$

At date 0 an investor chooses $\theta_{0}$ to maximize

$$
\inf _{m \in \mathcal{M}} \mathrm{E}_{m}\left[V_{1}\left(w_{0}+\theta_{0}\left(p_{1}\left(\tilde{s}_{1}\right)-p_{0}\right), \tilde{s}_{1}\right)\right]
$$

The value function at date 0 is defined as

$$
V_{0}\left(w_{0}\right)=\sup _{\theta_{0} \in \mathcal{R}} \inf _{m \in \mathcal{M}} \mathrm{E}_{m}\left[V_{1}\left(w_{0}+\theta_{0}\left(p_{1}\left(\tilde{s}_{1}\right)-p_{0}\right), \tilde{s}_{1}\right)\right]
$$

There is no requirement for the $m$ attaining the infimum to be the same at each date. On the contrary it is important to allow an investor to choose different models at each date to guarantee dynamic consistency.

\section{Equilibrium}

In equilibrium the representative investor holds the asset and consumes the liquidating dividend; i.e. $\tilde{p}_{3}=\tilde{d}$ and $\theta_{0}=\theta_{1}=\theta_{2}=1$.

I use dynamic programming to derive equilibrium stock prices. Specifically, the derivation in each period consists of four steps: (i) determine the set of conditional beliefs described by the set of models $\mathcal{M}$, (ii) for each price and portfolio solve for the conditional belief that minimizes expected utility, (iii) for each price choose a portfolio that maximizes expected utility with respect to the worst case scenario conditional belief determined in part (ii), (iv) find the price such that the optimal portfolio determined in part (iii) equals the market portfolio.

The equilibrium when the signal noise variance is known is provided in the next proposition. The proof is straightforward and thus omitted. 
Proposition 6 (Savage Benchmark). Fix $\left(\sigma_{1}, \sigma_{2}\right)$. Then

$$
\begin{aligned}
p_{2}\left(s_{2}\right) & =\mathrm{E}_{\sigma_{2}}\left[\tilde{d} \mid \tilde{s}_{2}=s_{2}\right]-\gamma \operatorname{Var}_{\sigma_{2}}\left[\tilde{d} \mid \tilde{s}_{2}=s_{2}\right] \\
p_{1}\left(s_{1}\right) & =\mathrm{E}_{\left(\sigma_{1}, \sigma_{2}\right)}\left[\tilde{d} \mid \tilde{s}_{1}=s_{1}\right]-\gamma \operatorname{Var}_{\left(\sigma_{1}, \sigma_{2}\right)}\left[\tilde{d} \mid \tilde{s}_{1}=s_{1}\right] . \\
p_{0} & =\mathrm{E}_{\left(\sigma_{1}, \sigma_{2}\right)}[\tilde{d}]-\gamma \operatorname{Var}_{\left(\sigma_{1}, \sigma_{2}\right)}[\tilde{d}] .
\end{aligned}
$$

The effects of ambiguous information on the conditional distribution of fundamentals and the derivation of the equilibrium stock price when investors are averse to ambiguous information is provided below. I proceed in two steps: (i) I determine and discuss the set of conditional beliefs at each date and (ii) I determine the equilibrium price of the market portfolio at each date.

\section{Beliefs}

At date 2 investors have ambiguous information about the fundamentals of the economy. Each model $m \in \mathcal{M}$ links this information to fundamentals and hence determines a conditional belief for $\tilde{d}$ given $s_{1}$ and $s_{2}$. The unconditional distribution of $\tilde{d}$ is known and $s_{2}$ is a sufficient statistic ( $l_{1}$ is irrelevant) and hence the set of conditional beliefs is determined by applying Bayes rule to each $l_{2} \in \mathcal{L}_{2} 25$ Specifically, standard normal-normal updating for each $\sigma_{2} \in\left[\sigma_{2 a}, \sigma_{2 b}\right]$ leads to

$$
\tilde{d} \mid \tilde{s}_{2}=s_{2} \sim \mathrm{N}\left(\bar{d}+\beta_{d \mid s_{2}}\left(s_{2}-\bar{d}\right), \sigma_{d}^{2}\left(1-\beta_{d \mid s_{2}}\right)\right), \quad \beta_{d \mid s_{2}}=\frac{\sigma_{d}^{2}}{\sigma_{d}^{2}+\sigma_{2}^{2}}
$$

Ambiguity about the informativeness of $\tilde{s}_{2}$ leads to ambiguity about the posterior mean and variance and hence to a family of conditional beliefs (see the discussion in Section 【).

At date 1 investors have ambiguous information and anticipate ambiguous information about the fundamentals of the economy. Specifically, every model $m \in \mathcal{M}$ determines a conditional belief for $\tilde{s}_{2}$ given $s_{1}$. The set of conditional beliefs is determined by applying Bayes rule to each $\left(l_{1}, l_{2}\right) \in\left(\mathcal{L}_{1} \times \mathcal{L}_{2}\right)$. Specifically, standard normal-normal updating for each $\left(\sigma_{1}, \sigma_{2}\right) \in\left[\sigma_{1 a}, \sigma_{1 b}\right] \times\left[\sigma_{2 a}, \sigma_{2 b}\right]$ leads to

$$
\tilde{s}_{2} \mid \tilde{s}_{1}=s_{1} \sim \mathrm{N}\left(\bar{d}+\beta_{s_{2} \mid s_{1}}\left(\tilde{s}_{1}-\bar{d}\right),\left(\sigma_{d}^{2}+\sigma_{2}^{2}\right)\left(1-\beta_{s_{2} \mid s_{1}}\right)\right),
$$

\footnotetext{
${ }^{25}$ See Appendix $\mathrm{F}$ for normal-normal updating rules.
} 
in which

$$
\beta_{s_{2} \mid s_{1}}=\frac{\sigma_{d}^{2}+\sigma_{2}^{2}}{\sigma_{d}^{2}+\sigma_{2}^{2}+\sigma_{1}^{2}}
$$

Ambiguity about the informativeness of $\tilde{s}_{1}$ and $\tilde{s}_{2}$ leads to ambiguity about the posterior mean and variance. Moreover, the posterior mean depends on the realization of $\tilde{s}_{1}$ and hence the worst case scenario belief will depend on $s_{1}$.

Suppose the investor has long position in the asset. The worst case scenario for the mean is when investors put more weight on bad news than on good news. Specifically,

$$
\min _{\substack{\sigma_{1} \in\left[\sigma_{1 a}, \sigma_{1 b}\right] \\ \sigma_{2} \in\left[\sigma_{2 a}, \sigma_{2 b}\right]}} \mathrm{E}_{\left(\sigma_{1}, \sigma_{2}\right)}\left[\tilde{s}_{2} \mid \tilde{s}_{1}=s_{1}\right]= \begin{cases}\bar{d}+\beta_{s_{2} \mid s_{1}}\left(\sigma_{1 b}, \sigma_{2 a}\right)\left(s_{1}-\bar{d}\right) & \text { if } \quad s_{1}-\bar{d}>0 \\ \bar{d}+\beta_{s_{2} \mid s_{1}}\left(\sigma_{1 a}, \sigma_{2 b}\right)\left(s_{1}-\bar{d}\right) & \text { if } \quad s_{1}-\bar{d}<0 \\ \bar{d} & \text { if } \quad s_{1}-\bar{d}=0 .\end{cases}
$$

The slope $\beta_{s_{2} \mid s_{1}}$, which measures how much weight to put on $s_{1}$ and how much information about $\tilde{s}_{2}$ is revealed by $s_{1}$, is strictly decreasing in the signal noise variance of the first signal but strictly increasing in the signal noise variance of the second signal because investors learn more from the current signal if there is more noise in the future signal.

The worst case scenario for the residual variance is a lot of noise in both signals. Specifically,

$$
\max _{\substack{\sigma_{1} \in\left[\sigma_{1 a}, \sigma_{1 b}\right] \\ \sigma_{2} \in\left[\sigma_{2 a}, \sigma_{2 b}\right]}} \operatorname{Var}_{\left(\sigma_{1}, \sigma_{2}\right)}\left[\tilde{s}_{2} \mid \tilde{s}_{1}=s_{1}\right]=\left(\sigma_{d}^{2}+\sigma_{2 b}^{2}\right)\left(1-\beta_{s_{2} \mid s_{1}}\left(\sigma_{1 b}, \sigma_{2 b}\right)\right) .
$$

At date 0 investors anticipate future ambiguous information about the fundamentals of the economy. Specifically, every model $m \in \mathcal{M}$ determines a belief for $\tilde{s}_{1}$. The set of beliefs is determined by applying Bayes rule to each $\left(l_{1}, l_{2}\right) \in\left(\mathcal{L}_{1} \times \mathcal{L}_{2}\right)$. Specifically, standard normal-normal updating for each $\left(\sigma_{1}, \sigma_{2}\right) \in\left[\sigma_{1 a}, \sigma_{1 b}\right] \times\left[\sigma_{2 a}, \sigma_{2 b}\right]$ leads to

$$
\tilde{s}_{1} \sim \mathrm{N}\left(\bar{d}, \sigma_{d}^{2}+\sigma_{2}^{2}+\sigma_{1}^{2}\right)
$$

Ambiguity about the informativeness of future signals leads to ambiguity about the posterior variance and thus to a family of beliefs at date zero even though there is no ex ante ambiguity about the dividend at date 3 . This concludes the derivation of the family of conditional 
beliefs at each date. I now proceed with the derivation of the equilibrium at each date.

\section{Equilibrium}

The set of models considered at date 2 leads to a set of conditional beliefs described by the interval $\left[\sigma_{2 a}, \sigma_{2 b}\right]$. Hence, the value function given in equation (35) is

$$
V_{2}\left(w_{2}, s_{2}\right)=\max _{\theta_{2} \in \mathcal{R}} \min _{\sigma_{2} \in\left[\sigma_{2 a}, \sigma_{2 b}\right]} \mathrm{E}_{\sigma_{2}}\left[u\left(w_{2}+\theta_{2}\left(\tilde{d}-p_{2}\right)\right) \mid \tilde{s}_{2}=s_{2}\right]
$$

The optimization problem at date 2 is the same as the static optimization problem discussed in the previous sections and hence the equilibrium signal-to-price map is provided in Theorem 126

Let $\mathrm{CE}_{2}\left(\theta_{2} ; w_{2}, p_{2}, s_{2}\right)$ denote the certainty equivalent of the ambiguity averse investor defined in equation (72) and determined in equation (12). Plugging in for the equilibrium price correspondence $p_{2}\left(\tilde{s}_{2}\right)$ leads to a unique equilibrium certainty equivalent correspondence. Specifically,

$$
\mathrm{CE}_{2}\left(s_{2}\right) \in\left\{\begin{array}{lll}
\left\{w_{2}+\frac{\gamma}{2} \operatorname{Var}_{\sigma_{2 b}}\left[\tilde{d} \mid \tilde{s}_{2}=s_{2}\right]\right\} & \text { if } & s_{2}>\hat{s}_{2} \\
\mathcal{C} \mathcal{E}_{2}\left(\hat{s}_{2}\right) & \text { if } & s_{2}=\hat{s}_{2} \\
\left\{w_{2}+\frac{\gamma}{2} \operatorname{Var}_{\sigma_{2 a}}\left[\tilde{d} \mid \tilde{s}_{2}=s_{2}\right]\right\} & \text { if } & s_{2}<\hat{s}_{2}
\end{array}\right.
$$

Specifically, $\mathrm{CE}_{2} \in \mathcal{C} \mathcal{E}_{2}\left(\hat{s}_{2}\right)$, if $\exists \sigma_{2} \in\left[\sigma_{2 a}, \sigma_{2 b}\right]$ such that

$$
\mathrm{CE}_{2}=w_{2}+\frac{\gamma}{2} \operatorname{Var}_{\sigma_{2}}\left[\tilde{d} \mid \tilde{s}_{2}=\hat{s}_{2}\right]
$$

This concludes the derivation at date 2 .

The set of models considered at date 1 leads to a set of conditional beliefs characterized by the two intervals $\left[\sigma_{1 a}, \sigma_{1 b}\right]$ and $\left[\sigma_{2 a}, \sigma_{2 b}\right]$. Hence, the value function given in equation (37) is

$$
V_{1}\left(w_{1}, s_{1}\right)=\max _{\theta_{1} \in \mathcal{R}} \min _{\left(\sigma_{1}, \sigma_{2}\right) \in\left[\sigma_{1 a}, \sigma_{1 b}\right] \times\left[\sigma_{2 a}, \sigma_{2 b}\right]} \mathrm{E}_{\left(\sigma_{1}, \sigma_{2}\right)}\left[u\left(\mathrm{CE}_{2}\left(\tilde{w}_{2}, \tilde{s}_{2}\right)\right) \mid \tilde{s}_{1}=s_{1}\right]
$$

in which $\tilde{w}_{2}=w_{1}+\theta_{1}\left(p_{2}\left(\tilde{s}_{2}\right)-p_{1}\right)$ and $p_{2}(\cdot)$ is given in equation (15) and $\mathrm{CE}_{2}(\cdot)$ is given

\footnotetext{
${ }^{26}$ Let $\hat{s}_{2}=\bar{d}-\gamma \sigma_{d}^{2} / 2, \beta_{2}=\beta_{d \mid s_{2}}\left(\sigma_{2}\right), \beta_{2 a}=\beta_{d \mid s_{2}}\left(\sigma_{2 b}\right)$, and $\beta_{2 b}=\beta_{d \mid s_{2}}\left(\sigma_{2 a}\right)$ in Theorem 1 .
} 
in equation (51). The price and the certainty equivalent are multi-valued at $\hat{s}_{2}$ but the probability of $\tilde{s}_{2}=\hat{s}_{2}$ is zero and hence the expectation in equation (52) is well defined.

To gain some more intuition consider first the case in which investors are risk neutral. In this case the price of the market portfolio is equal to the expected future stock price when the worst case scenario is used to evaluate the expected value. Specifically,

$$
p_{1}\left(s_{1}\right)=\min _{\left(\sigma_{1}, \sigma_{2}\right) \in\left[\sigma_{1 a}, \sigma_{1 b}\right] \times\left[\sigma_{2 a}, \sigma_{2 b}\right]} \mathrm{E}_{\left(\sigma_{1}, \sigma_{2}\right)}\left[p_{2}\left(\tilde{s}_{2}\right) \mid \tilde{s}_{1}=s_{1}\right]
$$

in which

$$
p_{2}\left(\tilde{s}_{2}\right)=\bar{d}+\beta_{2 b}\left(\tilde{s}_{2}-\bar{d}\right)-\left(\beta_{2 b}-\beta_{2 a}\right) \max \left(\tilde{s}_{2}-\bar{d}, 0\right)
$$

and hence

$$
\begin{aligned}
\mathrm{E}_{\left(\sigma_{1}, \sigma_{2}\right)}\left[p_{2}\left(\tilde{s}_{2}\right) \mid \tilde{s}_{1}=s_{1}\right] & =\bar{d}+\beta_{2 b} \mathrm{E}_{\left(\sigma_{1}, \sigma_{2}\right)}\left[\tilde{s}_{2}-\bar{d} \mid \tilde{s}_{1}=s_{1}\right] \\
& -\left(\beta_{2 b}-\beta_{2 a}\right) \mathrm{E}_{\left(\sigma_{1}, \sigma_{2}\right)}\left[\max \left(\tilde{s}_{2}-\bar{d}, 0\right) \mid \tilde{s}_{1}=s_{1}\right] .
\end{aligned}
$$

The investors knows that an ambiguous signal will arrive and hence requires an ambiguity premium to hold the asset. The ambiguity premium, which is given by the last term of equation (55), is determined in Proposition 7 of Appendix E, It is strictly increasing in the posterior mean and variance. Hence, ambiguity about the informativeness of both signals will affect the posterior mean and variance and thus the mean and ambiguity premium of the asset. Moreover, the mean and the ambiguity premium will depend on the realization of $\tilde{s}_{1}$.

The posterior variance dominates if the signals roughly confirms the unconditional mean of the divided $s_{1} \approx \bar{d}$ whereas the posterior mean dominates when the news are either very good or very bad $\left(\left|s_{1}-\bar{d}\right| \gg 0\right)$. Specifically, if the signal roughly confirms the unconditional mean of the dividend, then the worst case scenario is a large ambiguity premium. The ambiguity premium is large when the residual variance is large and hence both signals are treated as high noise. However, if the signal is either good $\left(\left|s_{1}-\bar{d}\right| \gg 0\right)$ or bad $\left(\left(s_{1} \ll \bar{d}\right)\right)$, then the worst case scenario is a low posterior mean. Hence, the current signal $\tilde{s}_{1}$ is treated as low noise for very bad news $\left(s_{1} \ll \bar{d}\right)$ and otherwise as high noise whereas the future signal $\tilde{s}_{2}$ is treated as low noise for very good news $\left(s_{1} \gg \bar{d}\right)$ and high 
noise otherwise 27

The next figure shows the equilibrium price as a function of the signal. Ambiguous information has two effects on the price of the market portfolio: (i) investors put more weight on bad than on good news and hence the price reacts more to bad news than to good news and (ii) the price of the asset is lower than the mean of the asset because investors anticipated that they will receive ambiguous information in the future and thus require a premium to hold the asset. Moreover, the mean and the ambiguity premium are continuous functions of the signal and hence the price of the market portfolio is a continuous and concave function of the signal.

\footnotetext{
${ }^{27}$ There are parameter values for which the worst case scenario signal noise distribution of either signal is attained in the interior of the signal noise interval for a small ranges of signal values. See Proposition 7 in Appendix E
} 


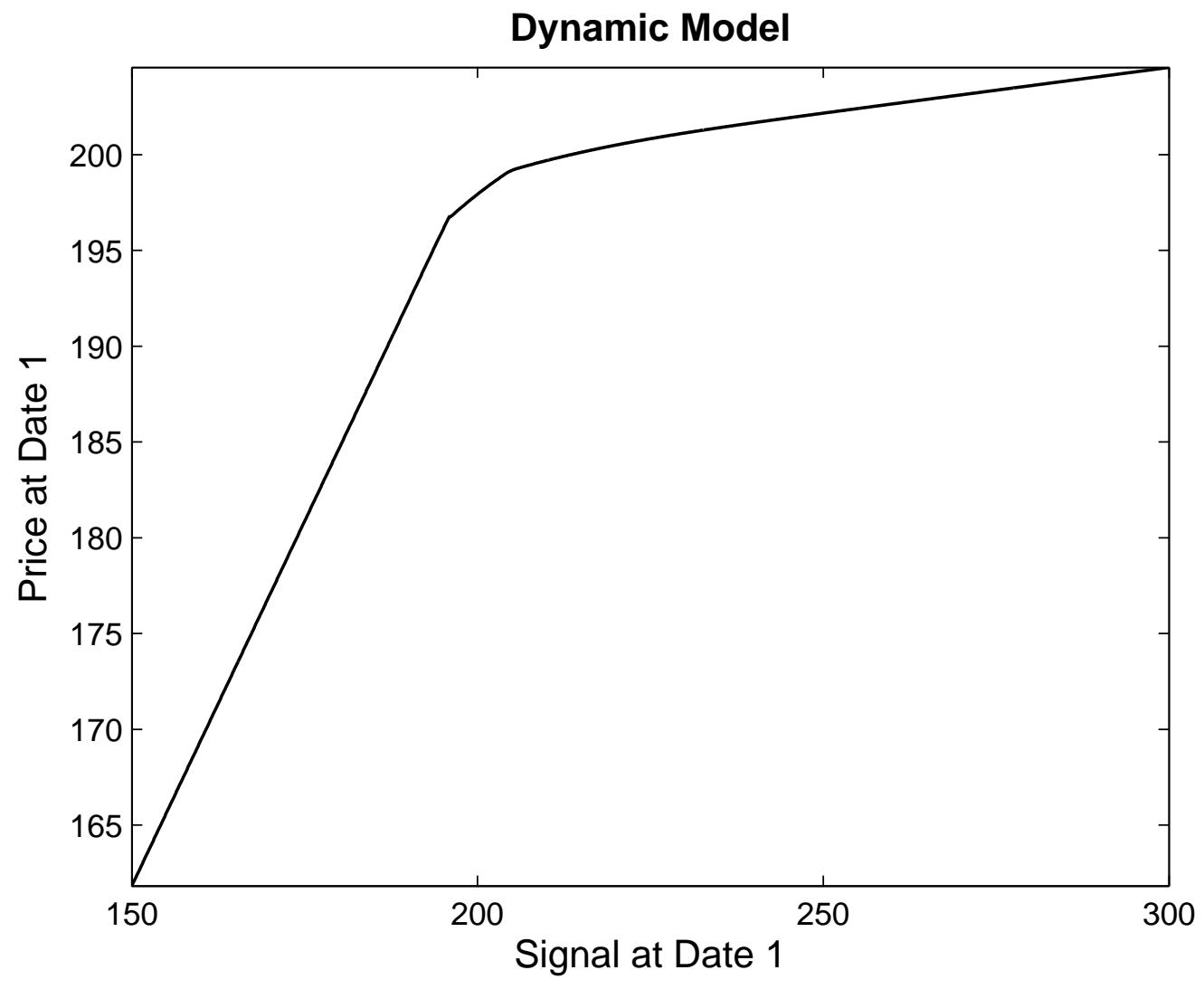

Figure 10: Equilibrium Signal-to-Price Map at Date 1

This figure shows the equilibrium signal-to-price map at date one when investors are risk neutral and ambiguity averse. The parameters are: $\bar{d}=200, \sigma_{d}=5$, and $\left[\sigma_{1 a}^{2}, \sigma_{1 b}^{2}\right]=$ $\left[\sigma_{1 a}^{2}, \sigma_{1 b}^{2}\right]=[1 / 3,3]$.

I solve for the equilibrium price numerically when investors are averse to risk and ambiguity. The worst case scenario for the representative investor depends on the realization of the current signal and the informativeness of the current and future signal. Specifically, I find that the representative investor treats $\tilde{s}_{1}$ as informative and $\tilde{s}_{2}$ as uninformative for bad news, $\tilde{s}_{1}$ as uninformative and $\tilde{s}_{2}$ as informative for good news, and both signals as uninformative for news that roughly confirm the unconditional mean of the dividend. Figure 11 shows the equilibrium signal to price map $p_{1}\left(s_{1}\right)$ for $\gamma=2$ and $\left[\sigma_{1 a}^{2}, \sigma_{1 b}^{2}\right]=\left[\sigma_{1 a}^{2}, \sigma_{1 b}^{2}\right]=$ $[1 / 3,3]$. 


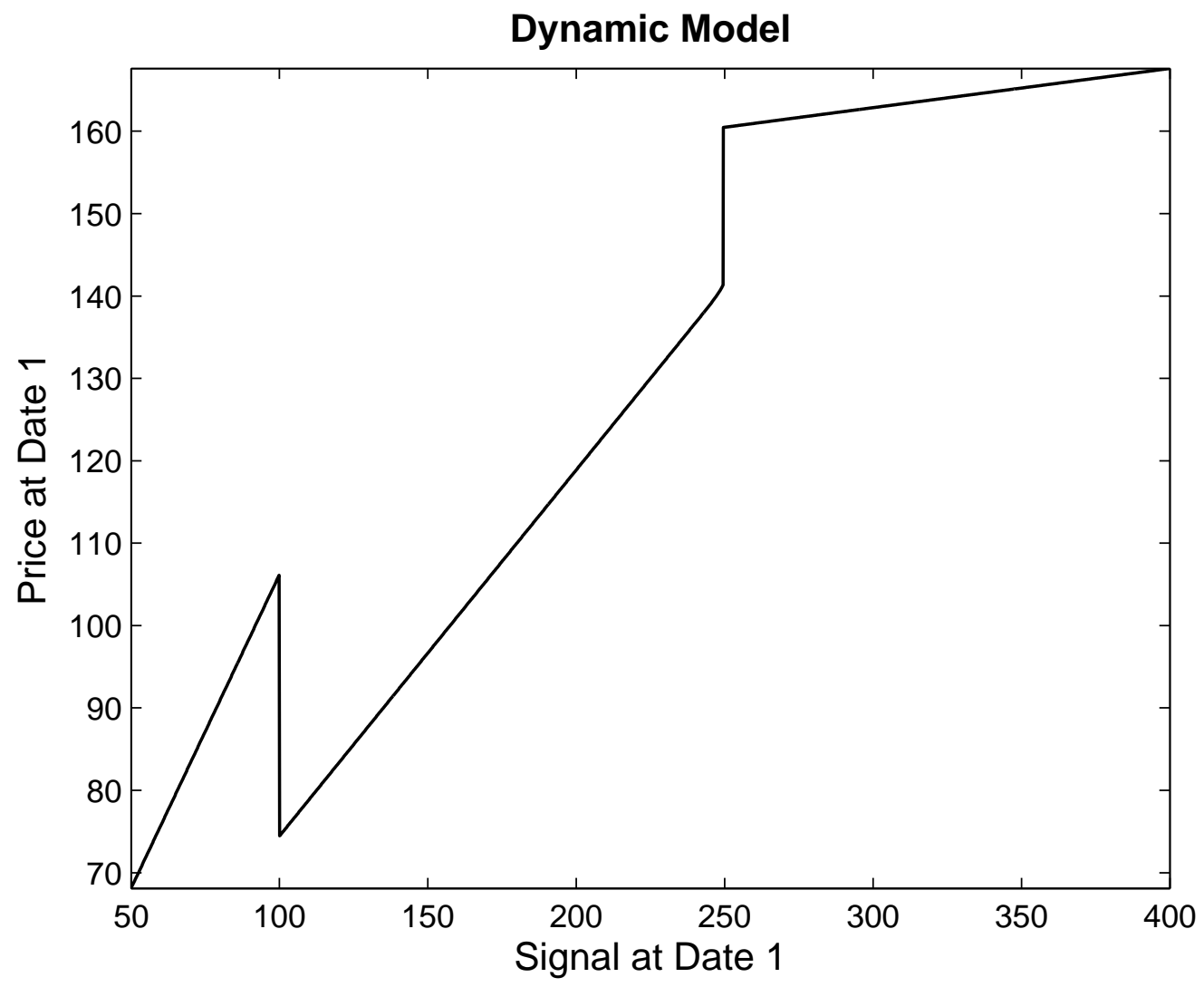

Figure 11: Equilibrium Signal-to-Price Map at Date 1

This figure shows the equilibrium signal-to-price map at date one. The parameters are: $\bar{d}=200, \sigma_{d}=5, \gamma=2$, and $\left[\sigma_{1 a}^{2}, \sigma_{1 b}^{2}\right]=\left[\sigma_{1 a}^{2}, \sigma_{1 b}^{2}\right]=[1 / 3,3]$.

Investors put more weight on bad than on good news and hence the price reacts more to bad news than to good news. Moreover, the price of the asset is lower than the mean of the asset because investors require a risk and ambiguity premium to hold the asset. If the signal roughly confirms the unconditional mean of the dividend, then the residual variance is larger than if the signal is very good or bad. Hence, if the signal increases trough the first critical signal value which is lower than the unconditional mean of the dividend, then the residual variance suddenly increases and hence the price drops. On the other had, if the signal increase trough the second critical value which is larger than the unconditional mean of the dividend, then the residual variance suddenly drops and hence the price increases.

The set of models considered at date 0 leads to a set of conditional beliefs characterized 
by the two intervals $\left[\sigma_{1 a}, \sigma_{1 b}\right]$ and $\left[\sigma_{2 a}, \sigma_{2 b}\right]$. Hence, the value function given in equation (39) is thus

$$
V_{0}\left(w_{0}\right)=\max _{\theta_{1} \in \mathcal{R}} \min _{\left(\sigma_{1}, \sigma_{2}\right) \in\left[\sigma_{1 a}, \sigma_{1 b}\right] \times\left[\sigma_{2 a}, \sigma_{2 b}\right]} \mathrm{E}_{\left(\sigma_{1}, \sigma_{2}\right)}\left[u\left(\mathrm{CE}_{1}\left(\tilde{w}_{1}, \tilde{s}_{1}\right)\right)\right]
$$

in which $\tilde{w}_{1}=w_{0}+\theta_{0}\left(p_{1}\left(\tilde{s}_{1}\right)-p_{0}\right)$ and $p_{1}(\cdot)$ and $\mathrm{CE}_{1}(\cdot)$ are to be determined numerically. The price and the certainty equivalent are multivalued at two critical signal values but the probability of attaining either of these two values is zero and hence the expectation in equation (56) is well defined 28

The price at date one is non-increasing and concave if the representative investor is risk neutral and hence the worst case scenario is a large signal noise of both signals. Specifically,

$$
\begin{aligned}
p_{0} & =\min _{\left(\sigma_{1}, \sigma_{2}\right) \in\left[\sigma_{1 a}, \sigma_{1 b}\right] \times\left[\sigma_{2 a}, \sigma_{2 b}\right]} \mathrm{E}_{\left(\sigma_{1}, \sigma_{2}\right)}\left[p_{1}\left(\tilde{s}_{1}\right)\right] \\
& =\mathrm{E}_{\left(\sigma_{1 b}, \sigma_{2 b}\right)}\left[p_{1}\left(\tilde{s}_{1}\right)\right]
\end{aligned}
$$

The numerical derivation of the price at date zero for risk averse investors is omitted.

\section{Conclusion}

In this paper I study the effects of aversion to risk and ambiguity on the value of the market portfolio when investors receive ambiguous information about fundamentals. I consider investors with CARA-utility who consider a set of models that consists of a single normally distributed marginal for fundamentals and a family of normally distributed conditionals that relate information to fundamentals. I show that when investors receive ambiguous information, then the interpretation of this information can change drastically. This leads to a discontinuity in the equilibrium-signal-to-price map. Moreover, the model can explain (i) excess volatility, (ii) negative skewness, and (iii) excess kurtosis of stock market returns.

\footnotetext{
${ }^{28}$ Suppose the two period model is extended to an N period model with signal structure $\tilde{s}_{n}=\tilde{s}_{n+1}+\tilde{\varepsilon}_{n+1}$, $\tilde{\varepsilon}_{n+1} \sim \mathrm{N}\left(0, \sigma_{n+1}^{2}\right)$, and $\sigma_{n} \in\left[\sigma_{a n}, \sigma_{n n}\right]$. Then it would still be the case that the minimization problem at each date would be at most two dimensional.
} 


\section{References}

Backus, David K., Bryan R. Routledge, and Stanley E. Zin, September 2004, Exotic preferences for macroeconomists, Working paper.

Bertsekas, Dimitri P., Angelia Nedić, and Asuman E. Ozdaglar, 2003, Convex Analysis and Optimization (Athena Scientific) 1 edn.

Billot, Antoine, Alain Chateuneuf, and Itzhak Gilboa, 2000, Sharing beliefs: Between agreeing and disagreeing, Econometrica 68, 685-694.

Brennan, Michael J., and Yihong Xia, 2001, Stock price volatility and equity premium, Journal of Monetary Economics 47, 249-283.

Caballero, Ricardo, and Arvind Krishnamurthy, 2008, Collective risk managment in a flight to quality episode, Journal of Finance LXIII, 2195-2230.

Cao, H. Henry, Tan Wang, and Harold H. Zhang, 2005, Model uncertainty, limited market participation, and asset prices, Review of Financial Studies 18, 1219-1251.

Chen, Zengjing, and Larry G. Epstein, 2002, Ambiguity, risk, and asset returns in continuous time, Econometrica 70, 1403-1443.

Dow, J., and S. Werlang, 1992, Uncertainty aversion, risk aversion, and the optimal choice of portfolio, Econometrica 60, 197-204.

Ellsberg, D., 1961, Risk, ambiguity, and the savage axioms, Quarterly Journal of Economics $75,643-69$.

Epstein, Larry G., and Martin Schneider, 2003, Recursive multiple-priors, Journal of Economic Theory 113, 1-31.

— , 2007, Learning under ambiguity, Review of Economic Studies 74, 1275-1303.

— 2008, Ambiguity, information quality, and asset pricing, Journal of Finance pp. $197-228$.

Epstein, Larry G., and Tan Wang, 1994, Intertemporal asset pricing under Knightian uncertainty, Econometrica 62, 283-322. 
Epstein, Larry G., and Stanley E. Zin, 1989, Substitution, risk aversion, and the temporal behavior of consumption and asset returns: A theoretical framework, Econometrica 57, 937-969.

Ergin, Haluk, and Faruk Gul, 2004, A subjective theory of compound lotteries, Working Paper.

Garlappi, Lorenzo, Raman Uppal, and Tan Wang, 2007, Portfolio selection with parameter and model uncertainty: A multi-prior approach, Review of Financial Studies 20, 41-81.

Gilboa, Itzhak, and David Schmeidler, 1989, Maxmin expected utility with non-unique prior, Journal of Mathematical Economics 18, 141-153.

Hansen, Lars Peter, and Thomas Sargent, 2001, Robust control and model uncertainty, American Economic Review 91, 60-66.

— 2007, Robustness (Princeton University Press) 1 edn.

Klibanoff, Peter, Massimo Marinacci, and Sujoy Mukerji, 2005, A smooth model of decison making under ambiguity, Econometrica 73, 1849-1892.

Knight, Frank H., 1921, Risk, Uncertainty, and Profit (Houghton Mifflin, Boston) 1 edn.

Kogan, Leonid, and Tan Wang, 2003, A simple theory of asset pricing under model uncertainty, Working paper.

Leippold, Markus, Fabio Trojani, and Paolo Vanini, 2008, Learning and asset prices under ambiguous information, Review of Financial Studies 21, 2565-2597.

LeRoy, Stephen F., and Richard D. Porter, 1981, The present-value relation: Test based on implied variance bounds, Econometrica 49, 555-574.

Maccheroni, Fabio, Massimo Marinacci, and Aldo Rustichini, 2006, Ambiguity aversion, robustness, and the variational representation of preferences, Econometrica 74, 14471498.

Maenhout, Pascal, 2004, Robust portfolio rules and asset pricing, Review of Financial Studies 17, 951-983. 
Mas-Colell, Andreu, Michael D. Whinston, and Jerry R. Green, 1995, Microeconomic Theory (Oxford University Press) 1 edn.

Nau, Robert F., 2006, Uncertainty aversion with second-order utilities and probabilities, Management Science 52, 136-145.

Rigotti, Luca, Chris Shannon, and Tomasz Strzalecki, 2008, Subjective beliefs and ex ante trade, Econometrica 76, 1167-1190.

Routledge, Bryan R., and Stanley E. Zin, 2001, Model uncertainty and liquidity, Working paper.

Savage, Leonard J., 1954, The foundation of statistics (Wiley, New York) 1 edn.

Schmeidler, David, 1989, Subjective probability and expected utility without additivity, Econometrica 57, 571-587.

Shiller, Robert, 1981, Do stock prices move too much to be justified by subsequent changes in dividends, American Economic Review 71, 421-436.

Veronesi, Pietro, 2000, How does information quality affect stock returns?, Journal of Finance pp. 807-837.

\section{A Convex Analysis}

In this section I provide some elementary results of Convex Analysis that I will use in the remainder of the appendix for all proofs. A good reference is the book by Bertsekas, Nedić, and Ozdaglar (2003).

Let $f: \mathcal{R} \rightarrow \mathcal{R}$ be a continuous function that is differentiable except for a finite number of points. Let $\mathcal{N}$ denote the finite set of points at which $f(x)$ is not differentiable.

Definition 2 (Left and Right Derivative of $f$ ). Let $f_{-}^{\prime}(\xi)$ denote the left derivative and $f_{+}^{\prime}(\xi)$ denote the right derivative of the function $f(x)$ evaluated at $x=\xi$. Specifically,

$$
\begin{aligned}
f_{+}^{\prime}(\xi) & \equiv \lim _{x \downarrow \xi} f^{\prime}(x) \\
f_{-}^{\prime}(\xi) & =\lim _{x \uparrow \xi} f^{\prime}(x) .
\end{aligned}
$$


Lemma 1. The function $f(x)$ is concave if (i) $f^{\prime \prime}(x) \leq 0 \forall x \in \mathcal{R} \backslash \mathcal{N}$ and (ii) $f_{-}^{\prime}(x) \geq f_{+}^{\prime}(x)$ $\forall x \in \mathcal{N}$.

Proof. Straightforward.

Definition 3 (Subgradient and Sudifferential). Let $f: \mathcal{R} \rightarrow \mathcal{R}$ be a concave function. The scalar $d \in \mathcal{R}$ is a subgradient of $f$ at a point $\xi \in \mathcal{R}$ if

$$
f(x) \leq f(\xi)+(x-\xi) d, \quad \forall x \in \mathcal{R}
$$

The set of all subgradients of a concave function $f$ at $\xi \in \mathcal{R}$ is called the subdifferential of $f$ at $\xi$ and is denoted by $\partial f(\xi)$.

The subdifferential of $f$ can be characterized by its right and left derivative. This is shown in the next Lemma.

Lemma 2 (Subdifferential of $f(x)$ ). Let $f: \mathcal{R} \rightarrow \mathcal{R}$ be a continuous and concave function. Then,

$$
\partial f(x)=\left\{d \mid f_{+}^{\prime}(x) \leq d \leq f_{-}^{\prime}(x)\right\}, \quad \forall x \in \mathcal{R} .
$$

Proof. See exercise 4.5 in Bertsekas, Nedić, and Ozdaglar (2003).

Necessary and sufficient conditions to obtain the maximum of $f$ are provided in the next lemma.

Lemma 3 (Maximum of $f(x)$ ). Let $f: \mathcal{R} \rightarrow \mathcal{R}$ be a continuous and concave function. Then,

$$
\xi \in \underset{x \in \mathcal{R}}{\operatorname{argmax}} f(x) \quad \text { if and only if } \quad 0 \in \partial f(\xi)
$$

Proof. Straightforward.

\section{B Ambiguous Information}

In this section I prove Proposition 1, I will proceed in four steps: (i) I determine $\mathrm{CE}(\cdot)$ in Lemma 4, (ii) then I discuss continuity and differentiability of $\mathrm{CE}(\cdot)$, (iii) then I derive 
the left and right derivative of $\mathrm{CE}(\cdot)$ at all points for which it is not differentiable, (iv) I conclude this section with the proof of Proposition 1 .

Let's define two functions for notional convenience: fix $\beta \in\left[\beta_{a}, \beta_{b}\right]$ and let $\phi_{\beta}(\theta) \equiv$ $\mathrm{CE}^{S}\left(\theta, \beta ; w_{0}, p, s\right)$ and fix $s \in \mathcal{R}$ and let $\psi_{s}(\theta)=\mathrm{CE}\left(\theta ; w_{0}, s, p\right)$.

Lemma 4. The certainty equivalent of the ambiguity averse investor is given by

$$
\begin{aligned}
\psi_{s}(\theta) & =w+(\bar{d}-p) \theta-\frac{1}{2} \gamma \sigma_{d}^{2} \theta^{2} \\
& +\max \left(s-\bar{d}+\frac{1}{2} \gamma \sigma_{d}^{2} \theta, 0\right)\left(\beta_{a} \max (\theta, 0)+\beta_{b} \min (\theta, 0)\right) \\
& +\min \left(s-\bar{d}+\frac{1}{2} \gamma \sigma_{d}^{2} \theta, 0\right)\left(\beta_{b} \max (\theta, 0)+\beta_{a} \min (\theta, 0)\right)
\end{aligned}
$$

Moreover, let $\hat{\theta} \equiv-2(s-\bar{d}) /\left(\gamma \sigma_{d}^{2}\right)$. Then,

$$
\psi_{s}(\theta)= \begin{cases}\phi_{\beta_{a}}(\theta) & \text { if } \theta \leq \min (\hat{\theta}, 0) \\ \phi_{\beta_{b}}(\theta) & \text { if } \min (\hat{\theta}, 0)<\theta<\max (\hat{\theta}, 0) \\ \phi_{\beta_{a}}(\theta) \quad \text { if } \theta \geq \max (\hat{\theta}, 0) .\end{cases}
$$

Proof. Plugging in for the conditional mean and variance in equation (8) leads to

$$
\phi_{\beta}(\theta)=w_{0}+(\bar{d}+\beta(s-\bar{d})-p) \theta-\frac{1}{2} \gamma \sigma_{d}^{2}(1-\beta) \theta^{2} .
$$

Let $\eta(\theta)=s-\bar{d}+\frac{1}{2} \gamma \sigma_{d}^{2} \theta$ and $g(\beta ; \theta)=\beta \eta(\theta)$. Hence,

$$
\phi_{\beta}(\theta)=w_{0}+(\bar{d}-p) \theta-\frac{1}{2} \gamma \sigma_{d}^{2} \theta^{2}+\theta g(\beta ; \theta)
$$

If $\theta$ is positive then $\phi_{\beta}(\theta)$ is minimized with respect to $\beta$ if $g(\beta, \theta)$ is minimized with respect to $\beta$ whereas if $\theta$ is negative then $\phi_{\beta}(\theta)$ is minimized with respect to $\beta$ if $g(\beta, \theta)$ is maximized with respect to $\beta$. Specifically,

$$
\psi_{s}(\theta)=w_{0}+(\bar{d}-p) \theta-\frac{1}{2} \gamma \sigma_{d}^{2} \theta^{2}+\max \{\theta, 0\} g_{\min }(\theta)+\min \{\theta, 0\} g_{\max }(\theta)
$$


in which

$$
\begin{aligned}
& g_{\min }(\theta) \equiv \min _{\beta \in\left[\beta_{a}, \beta_{b}\right]} g(\beta ; \theta)=\beta_{a} \max \{\eta(\theta), 0\}+\beta_{b} \min \{\eta(\theta), 0\} \\
& g_{\max }(\theta) \equiv \max _{\beta \in\left[\beta_{a}, \beta_{b}\right]} g(\beta ; \theta)=\beta_{b} \min \{\eta(\theta), 0\}+\beta_{a} \max \{\eta(\theta), 0\}
\end{aligned}
$$

Plugging $\eta(\theta), g_{\min }(\theta)$, and $g_{\max }(\theta)$ into equation (67) and rearranging terms leads to equation (63). The parameter $\hat{\theta}=-2(s-\bar{d}) /\left(\gamma \sigma_{d}^{2}\right)$ is the unique root of the function $\eta(\cdot)$, i.e. $\eta(\hat{\theta})=0$. Moreover, $\eta(\theta) \geq 0$ if $\theta \geq \hat{\theta}$ and $\eta(\theta)<0$ if $\theta<\hat{\theta}$. Evaluating equation (63) for the three different cases: (i) $s-\bar{d}=0 \Leftrightarrow \hat{\theta}=0$, (ii) $s-\bar{d}<0 \Leftrightarrow \hat{\theta}>0$, and (iii) $s-\bar{d}<0 \Leftrightarrow \hat{\theta}<0$ leads to equation (64).

Lemma 5. $\psi_{s}(\theta)$ is continuous $\forall \theta \in \mathcal{R}$. If $s=\bar{d}$, then $\psi_{s}(\theta)$ is continuously differentiable $\forall \theta \in \mathcal{R}$. If $s \neq \bar{d}$, then $\psi_{s}(\theta)$ is continuously differentiable $\forall \theta \in \mathcal{R} \backslash\{0, \hat{\theta}\}$.

Proof. Consider equation (63). Both the $\min (\cdot)$ and $\max (\cdot)$ function are continuous and hence $\psi_{s}(\theta)$ is a continuous function of $\theta$. If $s=\bar{d}$, then $\psi_{s}(\theta)=\phi_{\beta_{a}}(\theta)$ and hence is continuously differentiable.

The functions $\min (x, a)$ and $\max (x, a)$ are continuously differentiable expect for $x=a$. Hence, if $s \neq \bar{d}$, then $\psi_{s}(\theta)$ is continuously differentiable except when $\theta=0$ and $\theta=\hat{\theta}$. The latter follows directly from $\eta(\hat{\theta})=0$.

The right and left derivative of $\psi_{s}(\theta)$ are determined in the next lemma.

Lemma 6 (The right and left derivative of $\psi_{s}(\theta)$ ). Let $s \neq \bar{d}$. Then $\psi_{s}(\theta)$ is not differentiable at $\theta=0$ and $\theta=\hat{\theta}$. Moreover,

$$
\begin{aligned}
& \psi_{s}^{\prime+}(0)=\left\{\begin{array}{llc}
\bar{d}+\beta_{a}(s-\bar{d})-p & \text { if } & s-\bar{d}>0 \\
\bar{d}+\beta_{b}(s-\bar{d})-p & \text { if } & s-\bar{d}<0
\end{array}\right. \\
& \psi_{s}^{\prime-}(0)=\left\{\begin{array}{lll}
\bar{d}+\beta_{b}(s-\bar{d})-p & \text { if } & s-\bar{d}>0 \\
\bar{d}+\beta_{a}(s-\bar{d})-p & \text { if } & s-\bar{d}<0
\end{array}\right.
\end{aligned}
$$




$$
\begin{aligned}
& \psi_{s}^{\prime+}(\hat{\theta})=\left\{\begin{array}{lcc}
\bar{d}+2(s-\bar{d})-p-\beta_{b}(s-\bar{d}) & \text { if } & s-\bar{d}>0 \\
\bar{d}+2(s-\bar{d})-p-\beta_{a}(s-\bar{d}) & \text { if } & s-\bar{d}<0
\end{array}\right. \\
& \psi_{s}^{\prime-}(\hat{\theta})=\left\{\begin{array}{lll}
\bar{d}+2(s-\bar{d})-p-\beta_{a}(s-\bar{d}) & \text { if } & s-\bar{d}>0 \\
\bar{d}+2(s-\bar{d})-p-\beta_{b}(s-\bar{d}) & \text { if } & s-\bar{d}<0
\end{array}\right.
\end{aligned}
$$

Moreover, if $s=\bar{d}$, then $\hat{\theta}=0$ and

$$
\psi_{s}^{\prime+}(0)=\psi_{s}^{\prime-}(0)=\psi_{s}^{\prime}(0)=\bar{d}-p
$$

Proof. Taking the first derivative of $\phi_{\beta}(\theta)$ given in equations (8) and (65) leads to

$$
\begin{aligned}
\phi_{\beta}^{\prime}(\theta) & =\mathrm{E}_{\beta}[\tilde{d} \mid \tilde{s}=s]-p-\gamma \operatorname{Var}_{\beta}[\tilde{d} \mid \tilde{s}=s] \theta \\
& =\bar{d}+\beta(s-\bar{d})-p-\gamma \sigma_{d}^{2}(1-\beta) \theta .
\end{aligned}
$$

Consider three cases: (i) $s-\bar{d}=0 \Leftrightarrow \hat{\theta}=0$, (ii) $s-\bar{d}<0 \Leftrightarrow \hat{\theta}>0$, and (iii) $s-\bar{d}<0 \Leftrightarrow$ $\hat{\theta}<0$.

1. If $\hat{\theta}=0$, then $\psi_{s}(\theta)=\phi_{\beta_{a}}(\theta) \forall \theta \in \mathcal{R}$ and hence

$$
\psi_{s}(\hat{\theta})=\psi_{s}(0)=\psi_{s}^{+}(0)=\psi_{s}^{-}(0)=\bar{d}-p
$$

2. If $\hat{\theta}>0$, then taking the derivatives of $\psi_{s}(\theta)$ given in equation (64) with respect to $\theta$ except for $\theta=0$ and $\theta=\hat{\theta}$ leads to

$$
\psi_{s}^{\prime}(\theta)=\left\{\begin{array}{lll}
\phi_{\beta_{a}}^{\prime}(\theta) & \text { if } & \theta<0 \\
\phi_{\beta_{b}}^{\prime}(\theta) & \text { if } & 0<\theta<\hat{\theta} \\
\phi_{\beta_{a}}^{\prime}(\theta) & \text { if } & \theta>\hat{\theta} .
\end{array}\right.
$$

Moreover, taking the limit of $\psi_{s}^{\prime}(\theta)$ given in equation (77) at $\theta=\hat{\theta}$ from above leads to

$$
\begin{aligned}
\psi_{s}^{\prime+}(\hat{\theta}) & =\lim _{\theta \downarrow \hat{\theta}} \psi_{s}^{\prime}(\theta)=\lim _{\theta \downarrow \hat{\theta}} \phi_{\beta_{a}}^{\prime}(\theta) \\
& =\bar{d}+\beta_{a}(s-\bar{d})-p-\gamma \sigma_{d}^{2}\left(1-\beta_{a}\right) \hat{\theta} \\
& =\bar{d}-p+2(s-\bar{d})-\beta_{a}(s-\bar{d}) .
\end{aligned}
$$


Similarly, taking the limit of $\psi_{s}^{\prime}(\theta)$ at $\theta=\hat{\theta}$ from below leads to

$$
\begin{aligned}
\psi_{s}^{\prime-}(\hat{\theta}) & =\lim _{\theta \uparrow \hat{\theta}} \psi_{s}^{\prime}(\theta)=\lim _{\theta \uparrow \hat{\theta}} \phi_{\beta_{b}}^{\prime}(\theta) \\
& =\bar{d}+\beta_{b}(s-\bar{d})-p-\gamma \sigma_{d}^{2}\left(1-\beta_{b}\right) \hat{\theta} \\
& =\bar{d}-p+2(s-\bar{d})-\beta_{b}(s-\bar{d}) .
\end{aligned}
$$

Taking the limit of $\psi_{s}^{\prime}(\theta)$ given in equation (177) at $\theta=0$ from above leads to

$$
\begin{aligned}
\psi_{s}^{\prime+}(0) & =\lim _{\theta \downarrow 0} \psi_{s}^{\prime}(\theta)=\lim _{\theta \downarrow 0} \phi_{\beta_{b}}^{\prime}(\theta) \\
& =\bar{d}+\beta_{b}(s-\bar{d})-p .
\end{aligned}
$$

Similarly, taking the limit of $\psi_{s}^{\prime}(\theta)$ at $\theta=0$ from below leads to

$$
\begin{aligned}
\psi_{s}^{\prime-}(0) & =\lim _{\theta \uparrow 0} \psi_{s}^{\prime}(\theta)=\lim _{\theta \uparrow 0} \phi_{\beta_{a}}^{\prime}(\theta) \\
& =\bar{d}+\beta_{a}(s-\bar{d})-p .
\end{aligned}
$$

3. The proof for the case $\hat{\theta}<0$ is similar and thus omitted.

Proof of Proposition 1. I have determined CE $(\cdot)$ in Lemma 4 and I have shown in Lemma 6 that $\mathrm{CE}(\cdot)$ is continuous and continuously differentiable except for the critical values $\theta=0$ and $\theta=\hat{\theta}$ if $s \neq \bar{d}$.

It remains to show that $\psi_{s}(\theta)$ is concave. Both $\phi_{\beta_{a}}(\theta)$ and $\phi_{\beta_{b}}(\theta)$ are concave functions. Hence, it remains to show that the difference between the right derivative and the left derivative at $\theta=0$ and $\theta=\hat{\theta}$ (the points where both functions are connected but not differentiable) is negative (see Lemma 1).

Let $s-\bar{d}>0$, then

$$
\begin{aligned}
& \psi_{s}^{\prime+}(\hat{\theta})-\psi_{s}^{\prime-}(\hat{\theta})=-\left(\beta_{b}-\beta_{a}\right)(s-\bar{d})<0 \\
& \psi_{s}^{\prime+}(0)-\psi_{s}^{\prime-}(0)=-\left(\beta_{b}-\beta_{a}\right)(s-\bar{d})<0 .
\end{aligned}
$$


Similarly, let $s-\bar{d}<0$, then

$$
\begin{aligned}
& \psi_{s}^{\prime+}(\hat{\theta})-\psi_{s}^{\prime-}(\hat{\theta})=\left(\beta_{b}-\beta_{a}\right)(s-\bar{d})<0 \\
& \psi_{s}^{\prime+}(0)-\psi_{s}^{\prime-}(0)=\left(\beta_{b}-\beta_{a}\right)(s-\bar{d})<0
\end{aligned}
$$

If $s-\bar{d}=0$, then $\psi_{s}(\cdot)$ is concave because $\psi_{s}(\theta)=\phi_{\beta_{a}}(\theta) \forall \theta \in \mathcal{R}$.

\section{Portfolio Choice}

I have shown in Proposition 1 that $\operatorname{CE}\left(\theta ; w_{0}, p, s\right)=\psi_{s}(\theta)$ is a continuous and concave function of the stock demand $\theta$. Hence, if zero is contained in the subdifferential of $\mathrm{CE}\left(\theta^{*} ; w_{0}, p, s\right)$, then it follows from Lemma 2 that $\theta^{*}$ is a global maximum of $\operatorname{CE}\left(\theta ; w_{0}, p, s\right)$ for all $\theta \in \mathcal{R}$.

The subdifferential of $\operatorname{CE}\left(\theta ; w_{0}, p, s\right)$ is determined in the next lemma. The proof follows directly from Lemma 2 and Lemma 6 and is thus omitted.

Lemma 7 (Subdifferential of $\mathrm{CE}(\theta)$ ). If $s=\bar{d}$, then the subdifferential of $\psi_{s}(\theta)$ is singlevalued. Specifically,

$$
\partial \psi_{s}(\theta)=\left\{\phi_{\beta_{a}}^{\prime}(\theta)\right\}, \quad \forall \theta \in \mathcal{R}
$$

If $s \neq \bar{d}$, then the subdifferential is single valued except for $\theta=0$ and $\theta=\hat{\theta}$. Specifically,

$$
\partial \psi_{s}(\theta)=\left\{\psi_{s}^{\prime}(\theta)\right\} \quad \forall \theta \in \mathcal{R} \backslash\{0, \hat{\theta}\},
$$

in which

$$
\psi_{s}^{\prime}(\theta)= \begin{cases}\phi_{\beta_{a}}^{\prime}(\theta) & \text { if } \theta<\min (\hat{\theta}, 0) \\ \phi_{\beta_{b}}^{\prime}(\theta) & \text { if } \min (\hat{\theta}, 0)<\theta<\max (\hat{\theta}, 0) \\ \phi_{\beta_{a}}^{\prime}(\theta) & \text { if } \theta>\max (\hat{\theta}, 0) .\end{cases}
$$


The subdifferential is multi-valued at $\theta=0$ and $\theta=\hat{\theta}$. Specifically,

$$
\begin{aligned}
& \partial \psi_{s}(0)=\left\{d \mid \psi_{s}^{\prime+}(0) \leq d \leq \psi_{s}^{\prime-}(0)\right\} \\
& \partial \psi_{s}(\hat{\theta})=\left\{d \mid \psi_{s}^{\prime+}(\hat{\theta}) \leq d \leq \psi_{s}^{\prime-}(\hat{\theta})\right\}
\end{aligned}
$$

in which

$$
\begin{aligned}
& \psi_{s}^{\prime+}(0)=m_{\beta_{a}}(s) \mathcal{I}_{\{s \geq \bar{d}\}}+m_{\beta_{b}}(s) \mathcal{I}_{\{s \leq \bar{d}\}}-p \\
& =\bar{d}-p+\beta_{a} \max \{s-\bar{d}, 0\}+\beta_{b} \min \{s-\bar{d}, 0\} \\
& \psi_{s}^{\prime-}(0)=m_{\beta_{b}}(s) \mathcal{I}_{\{s \geq \bar{d}\}}+m_{\beta_{a}}(s) \mathcal{I}_{\{s \leq \bar{d}\}}-p \\
& =\bar{d}-p+\beta_{b} \max \{s-\bar{d}, 0\}+\beta_{a} \min \{s-\bar{d}, 0\} \\
& \psi_{s}^{\prime+}(\hat{\theta})=\left(m_{\beta_{b}}(s)-\hat{\theta} \lambda_{\beta_{b}}\right) \mathcal{I}_{\{s \geq \bar{d}\}}+\left(m_{\beta_{a}}(s)-\hat{\theta} \lambda_{\beta_{a}}\right) \mathcal{I}_{\{s \leq \bar{d}\}}-p \\
& =\bar{d}+2(s-\bar{d})-p-\beta_{b} \max \{s-\bar{d}, 0\}-\beta_{a} \min \{s-\bar{d}, 0\} \\
& \psi_{s}^{\prime-}(\hat{\theta})=\left(m_{\beta_{a}}(s)-\hat{\theta} \lambda_{\beta_{a}}\right) \mathcal{I}_{\{s \geq \bar{d}\}}+\left(m_{\beta_{a}}(s)-\hat{\theta} \lambda_{\beta_{a}}\right) \mathcal{I}_{\{s \leq \bar{d}\}}-p \\
& =\bar{d}+2(s-\bar{d})-p-\beta_{a} \max \{s-\bar{d}, 0\}-\beta_{b} \min \{s-\bar{d}, 0\},
\end{aligned}
$$

in which mathcal $I_{\{\cdot\}}$ is the indicator function and

$$
\begin{aligned}
m_{\beta}(s) & \equiv \mathrm{E}_{\beta}[\tilde{d} \mid \tilde{s}=s]=\bar{d}+\beta(s-\bar{d}) \\
\lambda_{\beta} & \equiv \gamma \operatorname{Var}_{\beta}[\tilde{d} \mid \tilde{s}=s]=\gamma \sigma_{d}^{2}(1-\beta) .
\end{aligned}
$$

Proof of Proposition 3. It follows from Lemma 3 that there is a unique maximum of the certainty equivalent of an ambiguity averse investor if and only if zero is contained in the subdifferential of the certainty equivalent. The certainty equivalent of an ambiguity averse investor is given in equation (12) and its subdifferential is provided in Lemma 7 .

Consider three cases: (i) $s-\bar{d}=0 \Leftrightarrow \hat{\theta}=0$, (ii) $s-\bar{d}>0 \Leftrightarrow \hat{\theta}<0$, and (iii) $s-\bar{d}<0 \Leftrightarrow$ $\hat{\theta}>0$.

The certainty equivalent is smooth in the first case and thus the proof is trivial.

Consider the case $s>\bar{d}$. The certainty equivalent is differentiable except for the critical 
points 0 and $\hat{\theta}<0$. Specifically,

$$
\psi_{s}^{\prime}(\theta)=\left\{\begin{array}{lll}
\phi_{\beta_{a}}^{\prime}(\theta)=m_{\beta_{a}}-p-\lambda_{\beta_{a}} \theta & \text { if } \quad \theta<\hat{\theta} \\
\phi_{\beta_{b}}^{\prime}(\theta)=m_{\beta_{b}}-p-\lambda_{\beta_{b}} \theta & \text { if } \quad \hat{\theta}<\theta<0 \\
\phi_{\beta_{a}}^{\prime}(\theta)=m_{\beta_{a}}-p-\lambda_{\beta_{a}} \theta & \text { if } \quad \theta>0 .
\end{array}\right.
$$

I first determine the range of prices at which the subdifferential of the certainty equivalent is single valued and equal to zero. Specifically, $\phi_{\beta_{a}}^{\prime}\left(\theta^{*}\right)=0$ implies that $\theta^{*}=\left(m_{\beta_{a}}(s)-p\right) / \lambda_{\beta_{a}}$. Hence, $\psi_{s}^{\prime}\left(\theta^{*}\right)=\phi_{\beta_{a}}^{\prime}\left(\theta^{*}\right)=0$ if and only if $\theta^{*}>0$ or $\theta^{*}<\hat{\theta}$. The range of prices for which this is true is $m_{\beta_{a}}(s)>p$ and $m_{\beta_{a}}(s)-\hat{\theta} \lambda_{\beta_{a}}<p$. Similarly, $\phi_{\beta_{b}}^{\prime}\left(\theta^{*}\right)=0$ implies that $\theta^{*}=\left(m_{\beta_{b}}(s)-p\right) / \lambda_{\beta_{b}}$. Hence, $\psi_{s}^{\prime}\left(\theta^{*}\right)=\phi_{\beta_{b}}^{\prime}\left(\theta^{*}\right)=0$ if and only if $\hat{\theta}<\theta^{*}<0$. The range of prices for which this is true is $m_{\beta_{b}}(s)<p$ and $m_{\beta_{b}}(s)-\hat{\theta} \lambda_{\beta_{b}}>p$. Moreover, $m_{\beta_{a}}(s)<m_{\beta_{b}}(s)$ and $m_{\beta_{b}}(s)-\hat{\theta} \lambda_{\beta_{b}}<m_{\beta_{a}}(s)-\hat{\theta} \lambda_{\beta_{a}}$ for all $\beta_{a}<\beta_{b}$ if $s>\bar{d}$.

It remains to determine the optimal demands if $m_{\beta_{a}}(s) \leq p \leq m_{\beta_{b}}(s)$ and if $m_{\beta_{b}}(s)-\hat{\theta} \lambda_{\beta_{b}} \leq$ $p \leq m_{\beta_{a}}(s)-\hat{\theta} \lambda_{\beta_{a}}$. I conjecture that (i) $\theta^{*}=0$ if and only if $m_{\beta_{a}}(s) \leq p \leq m_{\beta_{b}}(s)$ and (ii) $\theta^{*}=\hat{\theta}$ if and only if $m_{\beta_{b}}(s)-\hat{\theta} \lambda_{\beta_{b}} \leq p \leq m_{\beta_{a}}(s)-\hat{\theta} \lambda_{\beta_{a}}$.

The first conjecture is true if and only if the subdifferential of $\psi_{s}(\theta)$ contains zero when evaluated at $\theta=0$. Hence,

$$
0 \in \partial \psi_{s}(0) \Leftrightarrow \psi_{s}^{\prime+}(0) \leq 0 \wedge \psi_{s}^{\prime-}(0) \geq 0 \Leftrightarrow m_{\beta_{a}}(s)-p \leq 0 \wedge m_{\beta_{b}}(s)-p \geq 0 .
$$

The second conjecture is true if and only if the subdifferential of $\psi_{s}(\theta)$ contains zero when evaluated at $\theta=\hat{\theta}$. Hence,

$$
\begin{aligned}
0 \in \partial \psi_{s}(\hat{\theta}) & \Leftrightarrow \psi_{s}^{\prime+}(\hat{\theta}) \leq 0 \wedge \psi_{s}^{\prime-}(\hat{\theta}) \geq 0 \\
& \Leftrightarrow m_{\beta_{b}}(s)-\hat{\theta} \lambda_{\beta_{b}}-p \leq 0 \wedge m_{\beta_{a}}(s)-\hat{\theta} \lambda_{\beta_{a}}-p \geq 0 .
\end{aligned}
$$

The proof for the case $s<\bar{d}$ is similar and thus omitted. 


\section{Equilibrium}

Proof of Proposition 4. I know from Proposition 3 that the optimal demand of an investor with initial wealth $w_{h 0}$, risk aversion $\gamma_{h}$ and aversion to ambiguous information described by $\left[\beta_{a}, \beta_{b}\right]$ is

$$
\theta_{h}(p)= \begin{cases}\frac{\mathrm{E}_{\beta_{a}}[\tilde{d} \mid \tilde{s}=s]-p}{\gamma_{h} \operatorname{Var}_{\beta_{a}}[\tilde{d} \mid \tilde{s}=s]} & p \leq p_{1} \\ -\frac{2}{\gamma_{h} \sigma_{d}^{2}} \min (s-\bar{d}, 0) & p_{1}<p \leq p_{2} \\ \frac{\mathrm{E}_{\beta_{b}}[\tilde{d} \mid \tilde{s}=s]-p}{\gamma_{h} \operatorname{Var}_{\beta_{b}}[\tilde{d} \mid \tilde{s}=s]} & p_{2}<p \leq p_{3} \\ -\frac{2}{\gamma_{h} \sigma_{d}^{2}} \max (s-\bar{d}, 0) & p_{3}<p \leq p_{4} \\ \frac{\mathrm{E}_{\beta_{a}}[\tilde{d} \mid \tilde{s}=s]-p}{\gamma_{h} \operatorname{Var}_{\beta_{a}}[\tilde{d} \mid \tilde{s}=s]} & p>p_{4},\end{cases}
$$

with $p_{1}, \ldots, p_{4}$ given in equations (23), ., 26, respectively. The price levels $p_{1}, \ldots, p_{4}$ are the same for each investor and hence adding up the individual demands of all investor for all five intervals leads to the aggregate demand function

$$
\theta(p)=\sum_{h=1}^{H} \theta_{h}= \begin{cases}\frac{\mathrm{E}_{\beta_{a}}[\tilde{d} \mid \tilde{s}=s]-p}{\operatorname{Var}_{\beta_{a}}[\tilde{d} \mid \tilde{s}=s]} \sum_{h=1}^{H} \frac{1}{\gamma_{h}} & p \leq p_{1} \\ -\frac{2}{\sigma_{d}^{2}} \min (s-\bar{d}, 0) \sum_{h=1}^{H} \frac{1}{\gamma_{h}} & p_{1}<p \leq p_{2} \\ \frac{\mathrm{E}_{\beta_{b}}[\tilde{d} \mid \tilde{s}=s]-p}{\operatorname{Var}_{\beta_{b}}[\tilde{d} \mid \tilde{s}=s]} \sum_{h=1}^{H} \frac{1}{\gamma_{h}} & p_{2}<p \leq p_{3} \\ -\frac{2}{\sigma_{d}^{2}} \max (s-\bar{d}, 0) \sum_{h=1}^{H} \frac{1}{\gamma_{h}} & p_{3}<p \leq p_{4} \\ \frac{\mathrm{E}_{\beta_{a}}[\tilde{d} \mid \tilde{s}=s]-p}{\operatorname{Var}_{\beta_{a}}[\tilde{d} \mid \tilde{s}=s]} \sum_{h=1}^{H} \frac{1}{\gamma_{h}} & p>p_{4},\end{cases}
$$

which is the demand function of the representative investor with initial wealth $w_{0}=$ $\sum_{h=1}^{H} w_{0 h}$, risk aversion $\gamma=1 /\left(\sum_{h=1}^{H} 1 / \gamma_{h}\right)$, and ambiguity $\left[\beta_{a}, \beta_{b}\right]$.

Proof of Theorem 1. Take the optimal demand function given in equation (22) of Proposition 3 and set it equal to the market clearing stock demand of one. 
Consider four cases (i) $s=\hat{s}$, (ii) $s<\hat{s}$, (ii) $\hat{s}<s<0$ and (vi) $s \geq 0$ with $\hat{s}=\bar{d}-\gamma \sigma_{d}^{2} / 2$.

(i) if $s=\hat{s}$, then $\hat{\theta}=1$. The optimal demand $\theta(p)$ equals $\hat{\theta}=1=-2 \min (\hat{s}-d, 0) /\left(\gamma \sigma_{d}^{2}\right)$ if and only if $p_{1} \leq p \leq p_{2}$. Evaluating $p_{1}$ and $p_{2}$ at $\hat{s}$ leads to

$$
\begin{aligned}
& p_{1}=\mathrm{E}_{\beta_{a}}[\tilde{d} \mid s=\hat{s}]-\gamma \operatorname{Var}_{\beta_{a}}[\tilde{d} \mid s=\hat{s}] \\
& p_{2}=\mathrm{E}_{\beta_{b}}[\tilde{d} \mid s=\hat{s}]-\gamma \operatorname{Var}_{\beta_{b}}[\tilde{d} \mid s=\hat{s}]
\end{aligned}
$$

Hence, $\mathcal{P}(\hat{s})=\left[p_{1}, p_{2}\right]$ is the set of equilibrium prices. Moreover, it is straightforward to show that $p \in \mathcal{P}(\hat{s})$, if $\exists \beta \in\left[\beta_{a}, \beta_{b}\right]$ such that

$$
p=\mathrm{E}_{\beta}[\tilde{d} \mid \tilde{s}=\hat{s}]-\gamma \operatorname{Var}_{\beta}[\tilde{d} \mid \tilde{s}=\hat{s}] .
$$

If $s<\hat{s}$, then $\hat{\theta}>1$ and hence the market clearing stock demand is optimal and equal to one if and only if

$$
p(s)=\mathrm{E}_{\beta_{b}}[\tilde{d} \mid \tilde{s}=s]-\gamma \operatorname{Var}_{\beta_{b}}[\tilde{d} \mid \tilde{s}=s] .
$$

In this case the inequality

$$
p_{2}=\mathrm{E}_{\beta_{b}}[\tilde{d} \mid \tilde{s}=s]-\hat{\theta} \gamma \operatorname{Var}_{\beta_{b}}[\tilde{d} \mid \tilde{s}=s] \leq p \leq \mathrm{E}_{\beta_{b}}[\tilde{d} \mid \tilde{s}=s]=p_{3} .
$$

is always satisfied.

Similarly, if $\hat{s}<s<0$, then $\hat{\theta}<1$ and hence the market clearing stock demand is optimal and equal to one if and only if

$$
p(s)=\mathrm{E}_{\beta_{a}}[\tilde{d} \mid \tilde{s}=s]-\gamma \operatorname{Var}_{\beta_{a}}[\tilde{d} \mid \tilde{s}=s] .
$$

In this case the inequality

$$
p \leq p_{1}=\mathrm{E}_{\beta_{a}}[\tilde{d} \mid \tilde{s}=s]-\hat{\theta} \gamma \operatorname{Var}_{\beta_{a}}[\tilde{d} \mid \tilde{s}=s]
$$

is always satisfied.

Similarly, if $s \geq 0$, then $\hat{\theta} \leq 0$ and hence the market clearing stock demand is optimal and 
equal to one if and only if

$$
p(s)=\mathrm{E}_{\beta_{a}}[\tilde{d} \mid \tilde{s}=s]-\gamma \operatorname{Var}_{\beta_{a}}[\tilde{d} \mid \tilde{s}=s] .
$$

In this case the inequality

$$
p \geq p_{4}=\mathrm{E}_{\beta_{a}}[\tilde{d} \mid \tilde{s}=s]-\hat{\theta} \gamma \operatorname{Var}_{\beta_{a}}[\tilde{d} \mid \tilde{s}=s]
$$

is always satisfied. This concludes the proof

Proof of Proposition 5. Suppose there exists and equilibrium. Moreover, let $\left[\beta_{a}, \beta_{b}\right]=$ $\bigcap_{h=1}^{H}\left[\beta_{a h}, \beta_{b h}\right], \gamma=1 / \sum_{h=1}^{H}\left(1 / \gamma_{h}\right)$, and $\hat{s}=\bar{d}-\gamma \sigma_{d}^{2} / 2$. Define,

$$
\begin{aligned}
& p_{1}=\mathrm{E}_{\beta_{a}}[\tilde{d} \mid \tilde{s}=\hat{s}]+\frac{2}{\sigma_{d}^{2}} \operatorname{Var}_{\beta_{a}}[\tilde{d} \mid \tilde{s}=\hat{s}] \min (\hat{s}-\bar{d}, 0) \\
& p_{2}=\mathrm{E}_{\beta_{b}}[\tilde{d} \mid \tilde{s}=\hat{s}]+\frac{2}{\sigma_{d}^{2}} \operatorname{Var}_{\beta_{b}}[\tilde{d} \mid \tilde{s}=\hat{s}] \min (\hat{s}-\bar{d}, 0)
\end{aligned}
$$

Hence, investor h's optimal demand for the price range $p_{1} \leq p \leq p_{2}$ is constant. Specifically,

$$
\theta_{h}(p)=-\frac{2}{\gamma_{h} \sigma_{d}^{2}} \min (\hat{s}-\bar{d}, 0) \quad \forall p_{1} \leq p \leq p_{2}
$$

Summing over all investors leads to

$$
\sum_{h=1}^{H} \theta_{h}(p)=-\frac{2}{\sigma_{d}^{2}} \min (\hat{s}-\bar{d}, 0) \sum_{h=1}^{H} \frac{1}{\gamma_{h}}=1 \quad \forall p_{1} \leq p \leq p_{2} .
$$

If $\beta_{a}<\beta_{b}$, then $p_{1}<p_{2}$ and hence, there is an interval of equilibrium prices if investors share more than one belief. In this case $\mathcal{P}=\left[p_{1}, p_{2}\right]$ and it is straightforward to show that $p \in \mathcal{P}$, if $\exists \beta \in\left[\beta_{a}, \beta_{b}\right]$ such that

$$
p=\mathrm{E}_{\beta}[\tilde{d} \mid \tilde{s}=\hat{s}]-\gamma \operatorname{Var}_{\beta}[\tilde{d} \mid \tilde{s}=\hat{s}]
$$

If $\beta_{a}=\beta_{b}$, then $p(\hat{s})=p_{1} \leq p_{2}$, then there is a unique equilibrium price if investors share only one belief. 


\section{E Dynamic Model}

Proposition 7. The asset price at date one is a non-decreasing concave function of the signal. Specifically,

$$
\begin{aligned}
& p_{1}\left(s_{1}\right)=\min _{\left(\sigma_{1}, \sigma_{2}\right) \in\left[\sigma_{1 a}, \sigma_{1 b}\right] \times\left[\sigma_{2 a}, \sigma_{2 b}\right]} \mathrm{E}_{\left(\sigma_{1}, \sigma_{2}\right)}\left[p_{2}\left(\tilde{s}_{2}\right) \mid \tilde{s}_{1}=s_{1}\right] \\
& =\bar{d}+\beta_{2 b} \mathrm{E}_{\left(\sigma_{1}^{*}\left(s_{1}\right), \sigma_{2}^{*}\left(s_{1}\right)\right)}\left[\tilde{s}_{2}-\bar{d} \mid \tilde{s}_{1}=s_{1}\right]-\Lambda\left(\sigma_{1}^{*}\left(s_{1}\right), \sigma_{2}^{*}\left(s_{1}\right) ; s_{1}\right)
\end{aligned}
$$

in which

$$
\begin{aligned}
\Lambda\left(\sigma_{1}, \sigma_{2} ; s_{1}\right) & =\left(\beta_{2 b}-\beta_{2 a}\right) \mathrm{E}_{\left(\sigma_{1}, \sigma_{2}\right)}\left[\max \left(s_{2}-\bar{d}, 0\right) \mid \tilde{s}_{1}=s_{1}\right] \\
& =\left(\beta_{2 b}-\beta_{2 a}\right)\left(\sqrt{\frac{v\left(\sigma_{1}, \sigma_{2}\right)}{2 \pi}} e^{-\frac{1}{2} \frac{m\left(\sigma_{1}, \sigma_{2} ; s_{1}\right)^{2}}{v\left(\sigma_{1}, \sigma_{2}\right)}}+m\left(\sigma_{1}, \sigma_{2} ; s_{1}\right) N\left(\frac{m\left(\sigma_{1}, \sigma_{2} ; s_{1}\right)}{\sqrt{v\left(\sigma_{1}, \sigma_{2}\right)}}\right)\right)
\end{aligned}
$$

and $N(\cdot)$ denotes the cumulative distribution function of a standard normal distribution and $m\left(\sigma_{1}, \sigma_{2} ; s_{1}\right)$ and $v\left(\sigma_{1}, \sigma_{2}\right)$ denote the conditional mean and variance of $\tilde{s}_{2}-\bar{d}$ given $s_{1}$, respectively.

Moreover,

$$
\left(\sigma_{1}^{*}\left(s_{1}\right), \sigma_{2}^{*}\left(s_{1}\right)\right)= \begin{cases}\left(\sigma_{1 b}, \sigma_{2 a}\right) & \text { if } s_{1}<\hat{s}_{1 a} \\ \left(\bar{\sigma}_{1}, \sigma_{2 a}\right) & \text { if } \hat{s}_{1 a} \leq s_{1} \leq \hat{s}_{1 b} \\ \left(\sigma_{1 a}, \sigma_{2 a}\right) & \text { if } \hat{s}_{1 b}<s_{1}<\hat{s}_{1 c} \\ \left(\sigma_{1 a}, \bar{\sigma}_{2}\right) & \text { if } \hat{s}_{1 c} \leq s_{1} \leq \hat{s}_{1 d} \\ \left(\sigma_{1 a}, \sigma_{2 b}\right) & \text { if } s_{1}>\hat{s}_{1 d}\end{cases}
$$

It is straightforward to solve for $\bar{\sigma}_{1}, \bar{\sigma}_{2}, \hat{s}_{1 a}, \hat{s}_{1 b}, \hat{s}_{1 c}$, and $\hat{s}_{1 d}$ numerically. There are parameter values for which $\hat{s}_{1 a}>\hat{s}_{1 b}$ and $\hat{s}_{1 c}>\hat{s}_{1 d}$. In this case the minimum is always attained at the boundary.

Proof. I use Lemma 10 to determine the conditional expectation in equation (108). Then I write down the Karusch-Kuhn-Tucker conditions to solve for the minimum of the optimiza- 
tion problem

$$
\begin{array}{ll}
\min _{\sigma_{1}, \sigma_{2}} & \mathrm{E}_{\left(\sigma_{1}, \sigma_{2}\right)}\left[p_{2}\left(\tilde{s}_{2}\right) \mid \tilde{s}_{1}=s_{1}\right] \\
\text { s.t. } & \sigma_{1 a} \leq \sigma_{1} \leq \sigma_{1 b} \\
& \sigma_{2 a} \leq \sigma_{2} \leq \sigma_{2 b}
\end{array}
$$

\section{F Normal Distribution}

This appendix summarizes properties of normally distributed variables. The proofs are straightforward and hence omitted.

Lemma 8 (Normal-Normal Updating). Let

$$
\begin{array}{cc}
\tilde{d}=\bar{d}+\tilde{\varepsilon}_{d} & \tilde{\varepsilon}_{d} \stackrel{i d}{\sim} N\left(0, \sigma_{d}^{2}\right) \\
\tilde{s}_{2}=\tilde{d}+\tilde{\varepsilon}_{2} & \tilde{\varepsilon}_{2} \stackrel{i d}{\sim} N\left(0, \sigma_{2}^{2}\right) \\
\tilde{s}_{1}=\tilde{s}_{2}+\tilde{\varepsilon}_{1} & \tilde{\varepsilon}_{1} \stackrel{i d}{\sim} N\left(0, \sigma_{1}^{2}\right)
\end{array}
$$

The marginal distribution of $\tilde{s}_{2}$ and $\tilde{s}_{1}$ is normal. Specifically,

$$
\begin{aligned}
& \tilde{s}_{2} \sim N\left(\bar{d}, \sigma_{d}^{2}+\sigma_{2}^{2}\right) \\
& \tilde{s}_{1} \sim N\left(\bar{d}, \sigma_{d}^{2}+\sigma_{2}^{2}+\sigma_{1}^{2}\right) .
\end{aligned}
$$

The distribution of $\tilde{d}\left|s_{2}, \tilde{d}\right| s_{1}$, and $\tilde{s}_{2} \mid s_{1}$ is normal. Specifically,

$$
\begin{aligned}
\tilde{d} \mid s_{2} & \sim N\left(\bar{d}+\beta_{d \mid s_{2}}\left(s_{2}-\bar{d}\right), \sigma_{d}^{2}\left(1-\beta_{d \mid s_{2}}\right)\right) \\
\tilde{d} \mid s_{1} & \sim N\left(\bar{d}+\beta_{d \mid s_{1}}\left(s_{1}-\bar{d}\right), \sigma_{d}^{2}\left(1-\beta_{d \mid s_{1}}\right)\right) \\
\tilde{s}_{2} \mid s_{1} & \sim N\left(\bar{d}+\beta_{s_{2} \mid s_{1}}\left(s_{1}-\bar{d}\right),\left(\sigma_{d}^{2}+\sigma_{2}^{2}\right)\left(1-\beta_{s_{2} \mid s_{1}}\right)\right)
\end{aligned}
$$


with

$$
\begin{aligned}
& \beta_{d \mid s_{2}}=\frac{\operatorname{Var}[d]}{\operatorname{Var}\left[s_{2}\right]}=\frac{\sigma_{d}^{2}}{\sigma_{d}^{2}+\sigma_{2}^{2}} \\
& \beta_{d \mid s_{1}}=\frac{\operatorname{Var}[d]}{\operatorname{Var}\left[s_{1}\right]}=\frac{\sigma_{d}^{2}}{\sigma_{d}^{2}+\sigma_{2}^{2}+\sigma_{1}^{2}} \\
& \beta_{s_{2} \mid s_{1}}=\frac{\operatorname{Var}\left[s_{2}\right]}{\operatorname{Var}\left[s_{1}\right]}=\frac{\sigma_{d}^{2}+\sigma_{2}^{2}}{\sigma_{d}^{2}+\sigma_{2}^{2}+\sigma_{1}^{2}} .
\end{aligned}
$$

Lemma 9. Let $x$ denote a normally distributed variable. Specifically, $x \sim N\left(\mu, \sigma^{2}\right)$. Moreover, let $-\infty \leq a<b \leq \infty$ and $\alpha \in \mathcal{R}$. Then,

$$
\begin{aligned}
E & \equiv \mathrm{E}_{x}\left[e^{\alpha x} \mathbf{1}_{\{a \leq x \leq b\}}\right] \\
& =e^{\alpha \mu+\frac{1}{2} \alpha^{2} \sigma^{2}}\left(N\left(\frac{\mu+\alpha \sigma^{2}-a}{\sigma}\right)-N\left(\frac{\mu+\alpha \sigma^{2}-b}{\sigma}\right)\right) . \\
& =e^{\alpha \mu+\frac{1}{2} \alpha^{2} \sigma^{2}}\left(N\left(\frac{b-\left(\mu+\alpha \sigma^{2}\right)}{\sigma}\right)-N\left(\frac{a-\left(\mu+\alpha \sigma^{2}\right)}{\sigma}\right)\right) .
\end{aligned}
$$

Moreover,

$$
\begin{gathered}
\lim _{a \rightarrow-\infty} E=e^{\alpha \mu+\frac{1}{2} \alpha^{2} \sigma^{2}}\left(1-N\left(\frac{\mu+\alpha \sigma^{2}-b}{\sigma}\right)\right) \\
=e^{\alpha \mu+\frac{1}{2} \alpha^{2} \sigma^{2}} N\left(\frac{b-\left(\mu+\alpha \sigma^{2}\right)}{\sigma}\right), \\
\lim _{b \rightarrow \infty} E=e^{\alpha \mu+\frac{1}{2} \alpha^{2} \sigma^{2}} N\left(\frac{\mu+\alpha \sigma^{2}-a}{\sigma}\right) \\
=e^{\alpha \mu+\frac{1}{2} \alpha^{2} \sigma^{2}}\left(1-N\left(\frac{a-\left(\mu+\alpha \sigma^{2}\right)}{\sigma}\right)\right),
\end{gathered}
$$

and

$$
\lim _{\substack{a \rightarrow-\infty \\ b \rightarrow \infty}} E=e^{\alpha \mu+\frac{1}{2} \alpha^{2} \sigma^{2}} .
$$

Lemma 10. Let $x$ denote a normally distributed variable. Specifically, $x \sim N\left(\mu, \sigma^{2}\right)$. 
Moreover, let $-\infty \leq a<b \leq \infty$ and $\alpha \in \mathcal{R}$. Then,

$$
\begin{aligned}
E & \equiv \mathrm{E}_{x}\left[x e^{\alpha x} \mathbf{1}_{\{a \leq x \leq b\}}\right] \\
& =\frac{\sigma}{\sqrt{2 \pi}}\left(e^{a \alpha-\frac{1}{2}\left(\frac{\mu-a}{\sigma}\right)^{2}}-e^{b \alpha-\frac{1}{2}\left(\frac{\mu-b}{\sigma}\right)^{2}}\right) \\
& +\left(\mu+\alpha \sigma^{2}\right) e^{\alpha \mu+\frac{1}{2} \alpha^{2} \sigma^{2}}\left(N\left(\frac{\mu+\alpha \sigma^{2}-a}{\sigma}\right)-N\left(\frac{\mu+\alpha \sigma^{2}-b}{\sigma}\right)\right) . \\
& =\frac{\sigma}{\sqrt{2 \pi}}\left(e^{a \alpha-\frac{1}{2}\left(\frac{\mu-a}{\sigma}\right)^{2}}-e^{b \alpha-\frac{1}{2}\left(\frac{\mu-b}{\sigma}\right)^{2}}\right) \\
& +\left(\mu+\alpha \sigma^{2}\right) e^{\alpha \mu+\frac{1}{2} \alpha^{2} \sigma^{2}}\left(N\left(\frac{b-\left(\mu+\alpha \sigma^{2}\right)}{\sigma}\right)-N\left(\frac{a-\left(\mu+\alpha \sigma^{2}\right)}{\sigma}\right)\right) .
\end{aligned}
$$

Moreover,

$$
\begin{aligned}
\lim _{a \rightarrow-\infty} E & =-\frac{\sigma}{\sqrt{2 \pi}} e^{b \alpha-\frac{1}{2}\left(\frac{\mu-b}{\sigma}\right)^{2}}+\left(\mu+\alpha \sigma^{2}\right) e^{\alpha \mu+\frac{1}{2} \alpha^{2} \sigma^{2}} N\left(\frac{b-\left(\mu+\alpha \sigma^{2}\right)}{\sigma}\right) \\
\lim _{b \rightarrow \infty} E & =\frac{\sigma}{\sqrt{2 \pi}} e^{a \alpha-\frac{1}{2}\left(\frac{\mu-a}{\sigma}\right)^{2}}+\left(\mu+\alpha \sigma^{2}\right) e^{\alpha \mu+\frac{1}{2} \alpha^{2} \sigma^{2}} N\left(\frac{\mu+\alpha \sigma^{2}-a}{\sigma}\right) \\
\lim _{\substack{a \rightarrow-\infty \\
b \rightarrow \infty}} E & =\left(\mu+\alpha \sigma^{2}\right) e^{\alpha \mu+\frac{1}{2} \alpha^{2} \sigma^{2}}
\end{aligned}
$$

